\title{
Data assimilation of stratospheric constituents: a review
}

\author{
W. A. Lahoz ${ }^{1, *}$, Q. Errera ${ }^{2}$, R. Swinbank ${ }^{3}$, and D. Fonteyn ${ }^{2, * *}$ \\ ${ }^{1}$ Data Assimilation Research Centre, University of Reading, UK \\ ${ }^{2}$ BIRA-IASB, Brussels, Belgium \\ ${ }^{3}$ Met Office, Exeter, UK \\ *now at: Norsk Institutt for Luftforskning, NILU, Norway \\ ** now at: Belgian Federal Science Office, Belgium
}

Received: 30 May 2007 - Published in Atmos. Chem. Phys. Discuss.: 4 July 2007

Revised: 18 October 2007 - Accepted: 1 November 2007 - Published: 21 November 2007

\begin{abstract}
The data assimilation of stratospheric constituents is reviewed. Several data assimilation methods are introduced, with particular consideration to their application to stratospheric constituent measurements. Differences from meteorological data assimilation are outlined. Historically, two approaches have been used to carry out constituent assimilation. One approach has carried constituent assimilation out as part of a Numerical Weather Prediction system; the other has carried it out in a standalone chemical model, often with a more sophisticated representation of chemical processes. Whereas the aim of the Numerical Weather Prediction approach has been to improve weather forecasts, the aims of the chemical model approach have included providing chemical forecasts and analyses of chemical constituents. A range of constituent assimilation systems developed in these two areas is presented and strengths and weaknesses discussed. The use of stratospheric constituent data assimilation to evaluate models, observations and analyses, and to provide analyses of constituents, monitor ozone, and make ozone forecasts is discussed. Finally, the current state of affairs is assessed, future directions are discussed, and potential key drivers identified.
\end{abstract}

\section{Introduction}

In the 1990s, following years of development of meteorological data assimilation by the Numerical Weather Prediction (NWP) community, the data assimilation methodology (e.g. Kalnay, 2003) began to be applied to constituents (including aerosol), with a strong focus on stratospheric ozone (Rood, 2003, 2005). Because of its comparatively later application, constituent data assimilation is less mature than meteorological data (henceforth NWP) assimilation. Nev-

Correspondence to: W. A. Lahoz

(wal@nilu.no) ertheless, there has been substantial progress over the last 15 years, with the field evolving from initial efforts to test the methodology to later efforts focusing on products for monitoring ozone and other constituents. More recently, the production of ozone forecasts by a number of operational centres (e.g. the European Centre for Medium-range Weather Forecasts, ECMWF, Dethof, 2003) has become routine. A notable feature of the application of the data assimilation methodology to stratospheric constituents has been the strong interaction between the NWP and research communities, for example, in the EU-funded ASSET project (Lahoz et al., 2007).

The main aims for assimilating ozone in the stratosphere include the development of ozone and UV-forecasting capabilities; the need to monitor stratospheric ozone to track the evolution of the stratospheric composition, mainly ozone and the gases that destroy it (WMO, 2006), and assess compliance with the Montreal protocol; the need to evaluate the performance of instruments measuring ozone, especially those providing long-term datasets (e.g. TOMS, GOME); and improving skill in the stratosphere, chiefly through a better representation of stratospheric winds and temperature as a result of an improved representation of the stratospheric ozone distribution. The assimilation of ozone is also important for technical reasons, including: the constraints ozone observations provide on other constituents; the use of assimilation techniques to evaluate models and ozone observations; the development of computer code to assimilate instrument radiances sensitive to temperature and constituents; and the dynamical information provided by ozone tracer distributions. Other stratospheric constituents besides ozone that are of interest in this regard include $\mathrm{H}_{2} \mathrm{O}, \mathrm{N}_{2} \mathrm{O}, \mathrm{CH}_{4}, \mathrm{NO}_{2}, \mathrm{HNO}_{3}$, $\mathrm{ClO}, \mathrm{BrO}$ and aerosol (see IGACO, 2004 for a more complete list).

In NWP, the main motivation for stratospheric constituent assimilation has been the use of constituent information (in particular, water vapour and stratospheric ozone) to improve

Published by Copernicus Publications on behalf of the European Geosciences Union. 
the weather forecast. Historically, two approaches have been used for stratospheric constituent data assimilation. One has done assimilation as part of an NWP system, used for operational weather forecasting; the other has done assimilation in a standalone chemical model, either a chemical transport model (CTM) or a photochemical box model, often with a more sophisticated representation of chemical processes. Whereas the aim of the NWP approach has been to improve weather forecasts, the aims of the chemical model approach are broader, and include providing chemical forecasts and analyses of chemical constituents. In this review paper we will focus on these two approaches and compare their strengths and weaknesses. Illustrative examples of each approach will be provided.

Table 1 provides a summary of stratospheric chemistry satellite observations for the period 1978 to the present, that have been assimilated by NWP-based or chemical model data assimilation systems. References describing the satellites/instruments are provided.

The increasing maturity of data assimilation applied to stratospheric constituents, and increasing use of the methodology by the scientific community, means that a review of the field is timely. This review complements and builds on the recent review by Rood (2005) by focusing on two approaches to assimilate stratospheric constituents, NWP models and chemical models, providing examples and comparing and contrasting the two approaches. It also takes into account recent developments concerning in particular the use of data assimilation to evaluate the quality of observations and models associated with ozone and water vapour. This review summarizes in one publication and puts in context these later results.

In the remaining sections of this review we discuss the elements of data assimilation, with particular consideration to constituent data assimilation (Sect. 2). We then discuss NWP-based approaches to data assimilation (Sect. 3) and chemical model approaches to data assimilation (Sect. 4). We then discuss the evaluation of models, observations and analyses (Sect. 5), and provide examples of applications of stratospheric constituent data assimilation (Sect. 6). Finally, we assess the current state of affairs, discuss future directions and identify potential key drivers (Sect. 7). An Appendix lists acronyms used in this paper.

\section{Elements of data assimilation}

\subsection{Introduction}

Information on a system from observations based on geophysical measurements (the observed system) is discrete in both space and time, so that there are "information gaps". However, many applications require fully-specified geophysical fields. Thus, information needs to be mapped from measurement space (or observational space) to a state space (or model space), such as a (discretized) numerical model representation of the stratosphere. Although the models available for this mapping vary in their complexity, all models to some extent embody the physical laws that govern the observed system. Often, the model itself is said to embody the prior or background information on the observed system; however, the prior information can also represent a prior or background estimate of the observed system. The data assimilation (DA) problem aims to fill the "information gaps" in an optimal way; it can be stated, in non-mathematical terms, as: Find the best representation of the state of an evolving system given measurements made and prior information on the system, taking account of errors in the measurements and the prior information.

The observation operator transforms from the model space to the measurement space. It involves a mapping from geophysical inputs in model space (e.g. temperatures, constituent amounts) to simulate an instrument measurement in measurement space (e.g. radiances), taking into account the physics of the measurement and the characteristics of the instrument. The DA problem involves a minimization of the misfit between the model and the observations, and between the model and prior information to produce a solution referred to as the analysis. The role of the model operator, or the forward model, depends on the data assimilation approach (see Sect. 2.2). In the sequential approach, the model operator maps the analysis forward in time to give a background state for a subsequent assimilation cycle; in the variational approach, the model operator may be part of the analysis process.

In general the number of measurements $p$ is different (and usually smaller than) the dimension $n$ of the state space, making the DA problem ill-posed. Prior or background information is used to correct the ill-posed nature of the DA problem.

Although Bayesian estimation (Rodgers, 2000) defines a systematic and rigorous approach to data assimilation, its full-scale implementation in constituent data assimilation is impossible, chiefly due to the size of the problem. However, the Bayesian approach is still useful in that it provides general guidelines for developing a DA system and evaluating its results. Nevertheless, in many practical applications it is necessary to make drastic simplifying assumptions to the DA algorithm. Two main lines have been followed: (i) statistical linear estimation, and (ii) ensemble assimilation (Talagrand, 2003a).

Most standard DA algorithms, such as optimal interpolation, the Kalman filter and smoother, and variational methods, are built on statistical linear estimation. Another algorithm used, nudging, is an empirical forcing of the model fields toward the observed values, and can be described as an extremely simplified form of the Kalman filter. Bouttier and Courtier (1999) provide details of these algorithms.

Statistical linear estimation achieves Bayesian estimation when the system is linear and the errors are Gaussian. In particular, statistical linear estimation provides a way of 
Table 1. Summary of assimilated stratospheric chemistry satellite observations, 1978-present.

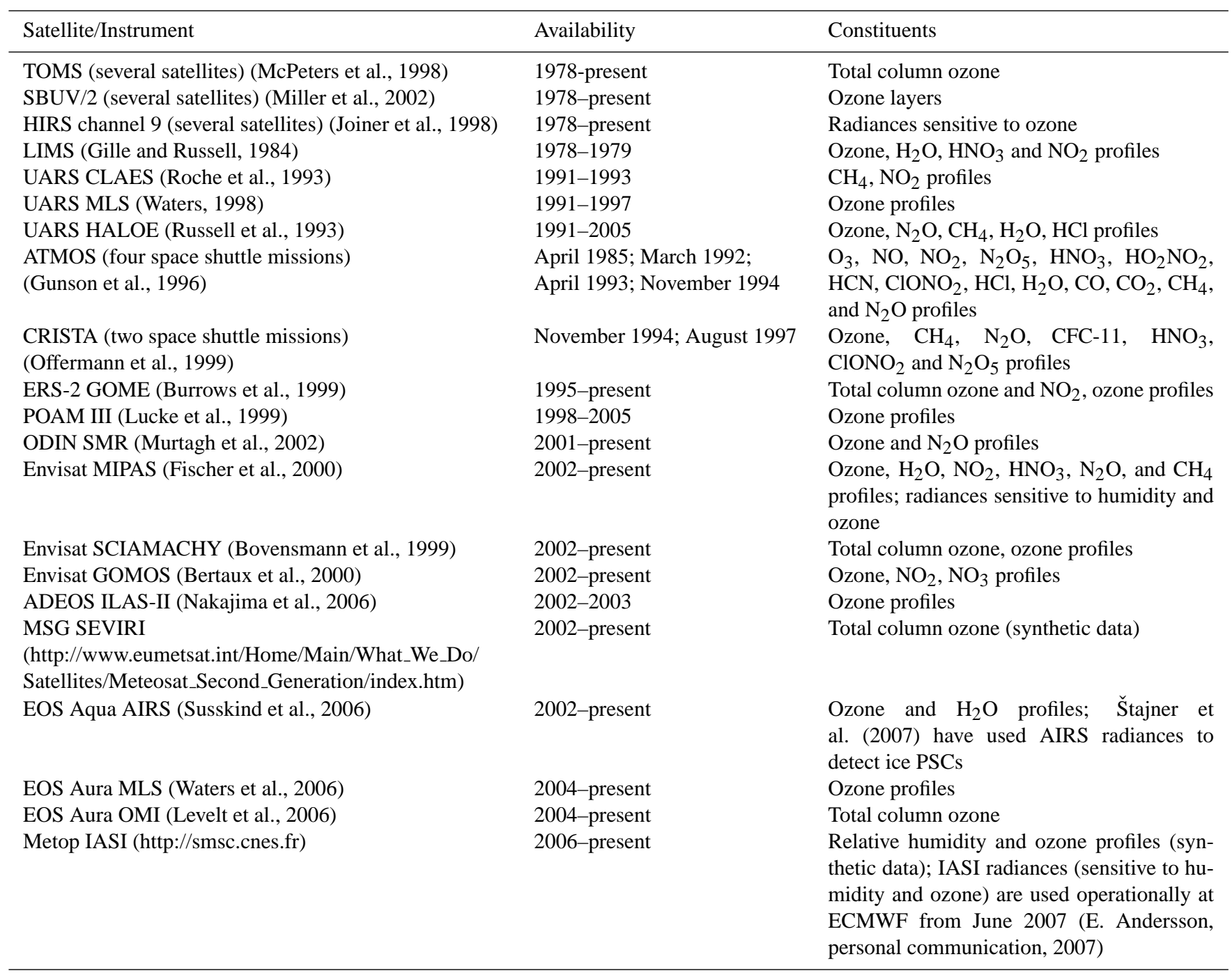

estimating the Best Linear Unbiased Estimate, BLUE (Talagrand, 2003a). Independently of the notion of statistical estimation, there exist two broad classes of numerical algorithms for data assimilation: variational and sequential (see Sect. 2.2). In the context of statistical linear estimation, these algorithms take respectively the form of the 4-D variational method (4D-Var), or the Kalman filter. (If the time dimension is omitted, the 4D-Var method becomes the 3-d variational method, 3D-Var.) These are two different algorithms for determining the BLUE, and they are equivalent under the only condition of linearity.

Ensemble assimilation is a form of Monte-Carlo approximation which attempts to estimate probability distribution functions (PDFs) from the spread of the ensemble. In present applications (e.g. the Ensemble Kalman filter, Evensen, 2003), the size of the analysed ensembles typically lies between a few tens to a few hundreds of model states.
There are differences between NWP and stratospheric constituent data assimilation that affect the way the assimilation is set up in the latter. These are:

- Stratospheric constituent data assimilation is less mature than NWP data assimilation. An example of this concerns parametrizations of ozone chemistry due to Cariolle and Déqué (1986). They have been used to assimilate ozone in the last 5 years or so, but it is only very recently that the performance of these schemes, and their associated errors, has been assessed in the data assimilation context (Geer et al., 2007).

- NWP is primarily an initial value problem. Stratospheric constituent data assimilation is commonly posed as an initial value problem, but sources and sinks may need to be considered. 
- Improvements in NWP can be achieved by more accurate specification of dynamical variables such as temperature, winds and humidity. For stratospheric constituents, a better forecast can be achieved both by a better description of dynamical variables (and hence transport of the constituent), and by a better description of sources and sinks (if applicable).

- The time-scales relevant for NWP are order of days. For stratospheric chemistry, there is a very wide range of time-scales, from decades (e.g. for carbon dioxide) to seconds for very short-lived species.

- Chemical equation systems are stiff, i.e., they include reactions with rates varying by several orders of magnitude. This requires the use of sophisticated numerical integration schemes, called stiff solvers. Stiffness manifests itself in strong error correlations between species, and can cause error covariance matrices to become singular. Constituent data assimilation algorithms must aim to account for these features.

- The availability of useful satellite observations of stratospheric composition is still relatively limited compared to the availability of observations of dynamical variables for NWP. Retrieval algorithms for stratospheric constituents are, however, reasonably well established, especially in comparison with the situation for tropospheric constituents.

- The Global Observing System for NWP is more mature than for constituents. This is reflected in that there are less operational instruments for constituents than for NWP. Many satellite constituent observations are classed as "research" or "pre-operational", which means that, compared to operational NWP observations, they are usually not available in near-real-time; the reliability of data supply is often less robust; and observational errors may be larger, or less well understood and characterized.

- For NWP the numerical dimension of the problem is extremely large; the typical dimension of current NWP models is of order $10^{7}$, while the number of observations available over $24 \mathrm{~h}$ is currently of order $10^{6}-10^{7}$. For stratospheric constituents, the number of data assimilated is generally an order of magnitude less than for NWP because fewer instruments are used, with fewer soundings per instrument. In both cases, however, the large dimension of the problem causes practical difficulties, influencing the practical implementation of assimilation systems.

- The dimensionality of the state of stratospheric chemical models is much higher than that of the NWP models. Assuming the same number of grid points, stratospheric constituent models typically need to follow between 20 and 100 different species, i.e., variables, per grid point, as compared to under a dozen variables for a NWP model.

One important difference between NWP and constituent data assimilation is worth emphasizing. In principle, given accurate initial conditions, sources and sinks and accurate dynamics, it would be possible to model constituent distributions many months without constituent data assimilation. Furthermore, in stratospheric chemistry, many situations can be modelled as a relaxation to an equilibrium state. This is very different to the chaotic system involved in dynamical data assimilation.

This does not mean that constituent data assimilation is unnecessary. Constituent data assimilation is needed to: (i) infer the constituent's initial conditions (we can only ever get these, imperfectly, from observations); (ii) correct for imperfectly known reaction rates; (iii) correct for imperfectly modelled chemistry (e.g. not enough species, not enough reactions described, or approximate parametrizations are needed); (iv) correct for unknown source terms (e.g. tropospheric pollution, troposphere-stratosphere transport); and (v) most importantly of all at the moment, correct for errors in constituent transport, such as excessive Brewer-Dobson circulations in analysed wind fields, or errors in temperature fields. Constituent data assimilation can thus be regarded as a way of providing accurate initial conditions (point (i)), and as a way of confronting models with observations in order to evaluate them and, in particular, correct model bias (points (ii)-(v)). The latter objective shows that constituent data assimilation is a different kind of problem compared to NWP data assimilation, where the goal is to get accurate initial conditions.

\subsection{Algorithms for constituent data assimilation}

The representation of errors is fundamental to the formulation of constituent DA algorithms. At its simplest one needs to consider the errors in the observations and the errors in the background information. Following Ide et al. (1997), R is the observation error covariance matrix. Typically, $\mathbf{R}$ is assumed to be diagonal; although this is not always justified (e.g. different elements of a retrieved profile are likely to have correlated errors). $\mathbf{R}$ includes the errors of the measurements themselves, E, and errors of representativeness, $\mathbf{F} ; \mathbf{R}=\mathbf{E}+\mathbf{F}$. $\mathbf{F}$ includes errors in the observation operator, and errors arising because the assimilation model does not fully resolve the scales measured by the observations (Cohn, 1997). B is the background error covariance matrix. Its offdiagonal elements determine how information is spread spatially from observation locations. If the background errors of one variable are uncorrelated with any other variable, then the analysis is termed univariate, but if the errors in different variables are correlated, the analysis is termed multivariate. If $\mathbf{B}$ is multivariate, it can provide statistical links between dynamical variables, for example, geostrophic coupling, or links between dynamical and chemical variables or different 
constituents. It is generally assumed that $\mathbf{B}$ and $\mathbf{R}$ are uncorrelated.

In general, in data assimilation, errors (for the observations and the background or model) are assumed to be Gaussian. The most fundamental justification for assuming Gaussian errors, which is entirely pragmatic, is the relative simplicity and ease of implementation of statistical linear estimation under these conditions. Because Gaussian PDFs are fully determined by their mean and their variance, the solution of the DA problem becomes computationally practical. Another argument for the choice of Gaussian errors is that of all possible PDFs with given mean and variance, the Gaussian distribution has maximum entropy (Rodgers, 2000).

Typically, there are biases between different observations types, and between the observations and the model. These biases are spatially and temporally varying, and it is a major challenge to estimate and correct them. Despite this, and mainly for pragmatic reasons, in stratospheric constituent data assimilation it is often assumed that the errors in the observations and the background or model are unbiased. For NWP, however, many assimilation schemes now incorporate a bias correction, which from the point of view of general estimation theory is the proper way to deal with biased data. For example, various techniques have been developed to correct observations to remove biases (e.g. Dee and da Silva, 1998). Dee (2005) reviews the treatment of biases in DA systems.

As described in Rood (2005), the data assimilation method adds an additional forcing to the equations of the model. Because of this, there is no reason to expect that correct geophysical/chemical balances are represented in an assimilated product. The products obtained from the model can be divided into primary and derived products. Primary products are quantities such as wind, temperature, water vapour and ozone, i.e., parameters most often explicitly modelled. Derived products are often functional relationships between the primary products, e.g., unobserved constituents. As discussed by Rood (2005), in a good DA system primary products tend to be well estimated. By contrast, derived products are likely to be physically or chemically inconsistent because of the forcing added by the DA method. Nevertheless, as shown later in this review, the DA method can provide reasonable estimates of derived products such as unobserved constituents. Molod et al. (1996) and Kistler et al. (2001) discuss the characteristics of errors associated with primary and derived products in DA systems.

We now describe variational and sequential methods in the context of constituent data assimilation, and then discuss some further aspects of the treatment of errors and observations.

Variational methods. In the 3 -d variational (3D-Var) method a minimization algorithm is used to find a model state, $\boldsymbol{x}$, that minimizes the misfit between $\boldsymbol{x}$ and the background state $\boldsymbol{x}^{b}$, and also between $\boldsymbol{x}$ and the observations $\boldsymbol{y}$. In $3 \mathrm{D}$-Var, we seek the minimum with respect to $\boldsymbol{x}$ of the penalty function, $J$ :

$J=\frac{1}{2}\left[\boldsymbol{x}-\boldsymbol{x}^{b}\right]^{T} \mathbf{B}^{-1}\left[\boldsymbol{x}-\boldsymbol{x}^{b}\right]+\frac{1}{2}[\boldsymbol{y}-H(\boldsymbol{x})]^{T} \mathbf{R}^{-1}[\boldsymbol{y}-H(\boldsymbol{x})]$.

The first term on the right-hand-side $\left(J_{b}\right)$ quantifies the misfit to the background term and the second term $\left(J_{o}\right)$ is the misfit to the observations. Extra terms incorporating dynamical constraints $\left(J_{c}\right)$ are also added in some implementations of 3D-Var. The observation operator $H$ maps the model state $\boldsymbol{x}$ to the measurement space, where $\boldsymbol{y}$ resides. If the observation operator is linear (written $\mathbf{H}$ ), the penalty function, $J$, is quadratic and is guaranteed to have a unique minimum. Among recent publications, Bouttier and Courtier (1999) discuss the solution of Eq. (1).

Because of the large number of variables involved, variational DA schemes do not perform the minimization of $J$ in the model space but, instead, use a transformed or control space. The elements of this control space are the control variables. A frequent choice of control variable for constituents is the logarithm of a normalized mixing ratio - this avoids unphysical negative values. It also means that errors can be specified as a proportion of the background value, rather than absolute concentrations, which is often more convenient since mixing ratios can vary by several orders of magnitude. However, such transformations can make the observation operator complex and non-linear, for example if the model state consists of local concentrations of a constituent while what is observed is an integral of concentrations along an instrument line of sight. Note that once a logarithm of the concentration is taken, the observation operator becomes non-linear, and that if one assumes Gaussian error statistics for concentrations, logarithms of concentrations will have non-Gaussian error statistics. It would also be possible to specify Gaussian errors of the logarithm of the concentrations.

$\mathbf{B}$ is generally built up by a series of control variable parameter and spatial transforms (Parrish and Derber, 1992; Lorenc et al., 2004). The control variables are chosen so that the structure of $\mathbf{B}$ is simplified, i.e., choosing control variables whose errors are (assumed to be) uncorrelated leads to a block diagonal matrix. Conversely, $\mathbf{B}$ can be set up in a way that explicitly couples constituent and dynamical fields where their errors are correlated, thus allowing the possibility of using tracer information to correct wind fields.

The 3D-Var algorithm assumes that all observations are valid at the same time, even though they are generally distributed over a time-window (of perhaps $6 \mathrm{~h}$ ). In 3D-FGAT (First Guess at the Appropriate Time), a variant of 3D-Var, the $J_{o}$ term is calculated by comparing observations with the background at the relevant observation times.

4-dimensional variational (4D-Var) assimilation is a development of 3D-Var in which the temporal dimension is included (e.g. Bouttier and Courtier, 1999). The minimization is carried out over a time window that is typically 6 or $12 \mathrm{~h}$, although longer time windows have been used. The natural length of the time window for diurnally varying species is 
$24 \mathrm{~h}$. In 4D-Var, observations are used at their correct time. Experiments at ECMWF suggest this is the main reason for the improved performance in 4D-Var, as compared to 3DVar (Fisher and Andersson, 2001). Experiments at the Met Office also indicate improved forecast skill for 4D-Var compared to an equivalent 3D-Var configuration (Rawlins et al., 2007). Thus, some of the benefit of $4 \mathrm{D}$-Var can be obtained using the 3D-FGAT approach.

4D-Var has two new features compared to 3D-Var. First, it includes a model operator, $M$, that carries out the evolution forward in time. The first derivative, or differential, of $M$, $\mathbf{M}$, is the tangent linear model (if $M$ is linear, represented by $\mathbf{M}$, its derivative is $\mathbf{M}$ ). The transpose of the tangent linear model operator, $\mathbf{M}^{T}$, integrates the adjoint variables backward in time. The tangent linear model is only defined under the condition that the function $J$ defined by Eq. (1) be differentiable - this is the tangent linear hypothesis (see Bouttier and Courtier, 1999, for further details). Second, $J$ can include an extra term in which the model errors associated with the model's temporal evolution are accounted for. For example, in the formulation of Zupanski (1997) an analogous term involving $\mathbf{Q}^{-1}$ is included in $J$, where $\mathbf{Q}$ is the model error covariance.

The properties of the adjoint method allow it to play two important roles in 4D-Var: coupling different elements of the algorithm, and computing gradients associated with the minimization of the penalty function (Talagrand, 2003b). The first property allows unobserved regions to be constrained by observed regions, this property being extended to unobserved species that chemically interact with modelled species that are observed; the second property allows efficient computation of the gradient of the penalty function.

Sequential methods. In the Kalman filter $(\mathrm{KF})$, a recursive sequential algorithm is applied to evolve a forecast, $\boldsymbol{x}^{f}$, and an analysis, $\boldsymbol{x}^{a}$, as well as their respective error covariance matrices, $\mathbf{P}^{f}$ and $\mathbf{P}^{a}$. The KF equations are (subscripts denote the timestep):

$\boldsymbol{x}_{n}^{f}=\mathbf{M}_{n-1} \boldsymbol{x}_{n-1}^{a} ;$

$\mathbf{P}_{n}^{f}=\mathbf{M}_{n-1} \mathbf{P}_{n-1}^{a} \mathbf{M}_{n-1}^{T}+\mathbf{Q}_{n-1} ;$

$\boldsymbol{x}_{n}^{a}=\boldsymbol{x}_{n}^{f}+\mathbf{K}_{n}\left[\boldsymbol{y}_{n}-\mathbf{H}_{n} \boldsymbol{x}_{n}^{f}\right]$;

$\mathbf{K}_{n}=\mathbf{P}_{n}^{f} \mathbf{H}_{n}^{T}\left[\mathbf{R}_{n}+\mathbf{H}_{n} \mathbf{P}_{n}^{f} \mathbf{H}_{n}^{T}\right]^{-1} ;$

$\mathbf{P}_{n}^{a}=\left[\mathbf{I}-\mathbf{K}_{n} \mathbf{H}_{n}\right] \mathbf{P}_{n}^{f}$.

Equation (2a) represents the forecast of the model fields from time-step $n-1$ to $n$, while (2b) calculates the forecast error covariance from the analysis error covariance $\mathbf{P}^{a}$ and the model error covariance $\mathbf{Q}$. Equations (2c) and (2e) are the analysis steps, using the Kalman gain defined in Eq. (2d). $\mathbf{Q}$ and $\mathbf{P}^{a}$ are assumed to be uncorrelated (e.g. Bouttier and Courtier, 1999). For optimality, all errors must be uncorrelated in time.
The Physical-space Statistical Analysis Scheme, PSAS (Cohn et al., 1998) consists in numerically solving Eq. (2c) by first computing the term $\boldsymbol{w}=\left[\mathbf{R}+\mathbf{H} \mathbf{P}^{f} \mathbf{H}^{T}\right]^{-1}\left[\boldsymbol{y}-\mathbf{H} \mathbf{x}^{f}\right]$ (we drop the subscript $n$ ) in observation space, and then multiplying $\boldsymbol{w}$ by $\mathbf{P}^{f} \mathbf{H}^{T}$, and adding the result to $\boldsymbol{x}^{f}$. This approach can be used to perform the updating step of the KF in Eq. (2c). PSAS (in its original 3-D form) can also be considered as the dual of 3D-Var, in which the analysis equation is solved in observation space, and then mapped to state space. The observation-space approach of PSAS is cheaper than the conventional model-space approach if the number of observations $p$ is much smaller than the dimension of the model state space $n$; this applies to many constituent assimilation applications. However, the relatively large value of $p$ in operational NWP systems means that the PSAS approach is less competitive than 3D-Var.

The KF can be generalized to non-linear $H$ and $M$ operators, although in this case neither the optimality of the analysis nor the equivalence with 4D-Var holds. The resulting equations are known as the Extended Kalman filter (EKF, Bouttier and Courtier, 1999). The cost of the KF or EKF is much larger than that of $4 \mathrm{D}$-Var, even with small models. This is a consequence of the explicit calculation of $\mathbf{P}^{f}$, and necessary storage costs. Consequently, development of $\mathrm{KF}$ techniques for constituent DA has tended to focus on approximate methods.

The Ensemble Kalman filter, EnKF, uses a Monte-Carlo ensemble of short-range forecasts to estimate $\mathbf{P}^{f}$. The estimation becomes more accurate as the ensemble size increases. The EnKF is more general than the EKF to the extent that it does not require validity of the tangent linear hypothesis. Evensen (2003) provides a comprehensive review of the theory and numerical implementation of the EnKF. Pham (2001) compares the EnKF and the particle filter. Recent advances include the square-root filter (Anderson, 2001) and local Ensemble Kalman filtering (Ott et al., 2004). To our knowledge, the EnKF is not currently used for stratospheric constituent assimilation. Note, however, that it has been used for tropospheric constituent data assimilation: for example, van Loon et al. (2000) have used it to assimilate ground-level ozone observations.

Treatment of errors. Many DA systems use the so-called National Meteorological Center, NMC, method (Parrish and Derber, 1992) to estimate the background error covariance matrix $\mathbf{B}$; this is based on the premise that forecast errors are similar to the differences between pairs of forecasts that verify at the same time. Polavarapu et al. (2005a) implement a variation on the NMC method in which $\mathbf{B}$ is estimated from successive $6 \mathrm{~h}$ differences from an extended model run. An alternative approach (Fisher, 2003) uses the spread from an ensemble of DA systems to estimate $\mathbf{B}$. To simplify the DA system and make it easier to assess the quality of observations, a simple approach is sometimes used with chemical models. As an example, Errera and Fonteyn (2001) assume a diagonal $\mathbf{B}$ (which is not valid) and increase the diagonal 
terms to compensate for the neglect of the off-diagonal terms.

To account for anisotropic atmospheric flow, flow dependence can be included in B (Riishøjgaard, 1998; Weaver and Courtier, 2001). Because anisotropy can impact tracer distributions, a flow-dependent $\mathbf{B}$ is sometimes used for the assimilation of stratospheric constituents: methane (Auger and Tangborn, 2004); ozone (Štajner et al., 2001; Segers et al., 2005). In the Auger and Tangborn set-up, the error covariances are evolved as a truncated set of wavelet coefficients; the truncation is carried out in such a way that the resolution of the error covariance is reduced only in the zonal direction, where methane gradients are smaller. In the Štajner et al. set-up, the correlation lengths are longer in the longitudinal than in the meridional direction, to match the distribution of SBUV/2 and TOMS ozone observations. In the Segers et al. set-up, the anisotropy is in all spatial directions, accounting for different correlation lengths for ozone with respect to height, latitude and longitude.

Theoretical work by Cohn (1993) demonstrated that background errors evolve according to advective dynamics. Swinbank et al. (2000) used this property to specify background errors using a series of Lagrangian back trajectories ending at the analysis time; the error correlations are specified in terms of the distance between trajectories $24 \mathrm{~h}$ earlier, so reflecting anisotropy developed over the previous day. Recent work by I. Stajner and colleagues at the Global Modeling and Assimilation Office (GMAO) (I. Štajner, personal communication, 2006) has developed the technique further and demonstrated small improvements in the areas not strongly constrained by observations or chemistry: lower stratosphere, near polar night, and troposphere. Another approach used is to implement a flow-dependent $\mathbf{B}$ based on the conservation properties of potential vorticity (Fierli et al., 2002).

Error covariance matrices in KF methods are parametrized in constituent DA to reduce cost; this approach to approximate the KF is referred to variously as the reduced, suboptimal or modified Kalman filter. $\mathbf{P}^{f}$ can be constructed by computing the diagonal elements and parametrizing the offdiagonal elements using adjustable parameters for the correlation lengths (Ménard et al., 2000; Ménard and Chang, 2000; Khattatov et al., 2000). Q can be specified by assuming that diagonal elements are proportional to the modelled field itself; they are used to update the diagonal elements of $\mathbf{P}^{f}$. This approach results in substantial savings, and allows the off-diagonal elements to be computed using a simple relation.

In both KF methods and 4D-Var, all model fields (dynamical and chemical) are implicitly coupled via the model operator M. This coupling is irrespective of whether the background error $\mathbf{B}$ (or forecast error $\mathbf{P}^{f}$ ) is formulated in a multivariate or univariate manner. In DA schemes the ozone control variable is often univariate (Errera and Fonteyn, 2001; Struthers et al., 2002), but it is still possible for constituent data to affect dynamical fields via $\mathbf{M}$ in 4D-Var and KF methods, although the coupling may be weak.
Treatment of observations. Observations are commonly divided into conventional observations (e.g. ground-based measurements and radiosondes) and satellite observations. Whereas conventional observations are often synoptic, meaning that all observations are taken at the same time, most observations of stratospheric constituents are from satellites, and are asynoptic. It is common in NWP (and relevant to constituent data assimilation) to reduce the amount of satellite data prior to assimilation. This process is called thinning and is done for two main reasons: to reduce computational cost and to ensure the density of data assimilated is consistent with the model length scales. The thinning of satellite observations also has the effect of reducing the spatial correlation in observation errors.

Satellites do not measure directly constituents; instead they measure photon counts (level 0 data). Radiative transfer algorithms then transform level 0 data into radiances (level 1 data). Subsequently, using inverse modelling techniques (Rodgers, 2000), height-resolved data or total column data of geophysical quantities (level 2 data) are inferred from level 1 data. Level 2 data are often termed retrievals. A recent development in inferring constituent retrievals has been the use of tomographic techniques to get a 2-D slice of the atmosphere, as opposed to the 1-D column provided by earlier methods (Carlotti et al. 2001).

Generally, constituent data are assimilated as retrievals. A recent development in the assimilation of constituents has been the assimilation of radiances sensitive to humidity and ozone from limb-sounding instruments measuring in the infrared (Bormann et al., 2005, 2007; Bormann and Healy, 2006; Bormann and Thépaut, 2006). Other efforts involving the assimilation of radiances sensitive to ozone include the use of HIRS channel 9 radiances at the Met Office (Jackson and Saunders, 2002; Jackson 2004), and the use of SBUV/2 radiances at the GMAO (Müller et al., 2004).

\section{NWP-based approaches}

\subsection{Introduction}

An NWP model is a complex numerical model designed to simulate the evolution of the atmospheric state over the length of a weather forecast (typically for a few hours up to two weeks into the future). The dynamical core of the model is concerned with solving the Navier-Stokes equations (or an approximation thereto) that govern the evolution of atmospheric winds, temperature and mass fields. The NWP dynamical core must solve for humidity, as the Navier-Stokes equations are formulated with moisture terms included. This means that mature humidity DA code has already been developed in operational NWP systems. Additional stratospheric humidity DA efforts must build on this code without unduly interfering with the assimilation of tropospheric humidity data. Details are provided in Sect. 3.2. 
The equations are typically solved using finite difference or spectral methods. Numerical models include parametrizations of a range of atmospheric physical processes, including the formation of clouds, production of rainfall, interactions of the flow with orography and radiative transfer processes, and, increasingly, chemistry.

There is a strong common heritage linking NWP models with general circulation models (GCMs) used for global climate simulations (e.g. Trenberth, 1992). In some cases, the same basic model is run in different configurations for both NWP and climate simulations (e.g. the Met Office Unified Model; Davies et al., 2005). The most complex atmospheric GCMs are coupled with sophisticated models of the ocean and land surface, to form Earth System Models.

Examples of NWP models used for constituent data assimilation include the National Centers Environmental Prediction, NCEP, system (see later); the ECMWF model, where ozone has been assimilated for analyses and forecasts (Dethof, 2003) and re-analyses (Dethof and Hólm, 2004), and where research has been done on the assimilation of limb infrared radiances sensitive to ozone and humidity (Bormann et al., 2005, 2007; Bormann and Healy, 2006; Bormann and Thépaut, 2007); and the Met Office model, where ozone has been assimilated for research (Jackson and Saunders, 2002; Struthers et al., 2002; Jackson, 2004, 2007; Lahoz et al., 2005, 2007; Geer et al., 2006a, b, 2007). Polavarapu et al. (2005a, b), using the Canadian middle atmosphere model (CMAM), discuss the role of dynamics on analysed stratospheric constituents, including ozone. As will be discussed in Sect. 3.3, in the NWP-based approach the use of simplified chemistry is the norm. An exception is the CMAM model, where full chemistry is used. The other main stratospheric constituent that has been assimilated into NWP models is water vapour (e.g. Lahoz et al., 2007). Table 2 provides a summary of selected data assimilation experiments based on NWP models and GCMs.

Hereafter we discuss the assimilation of stratospheric water vapour, and the stratospheric constituent that has received most attention over the past decade, ozone (Rood, 2003, 2005).

\subsection{Assimilation of humidity}

Water vapour is assimilated in the troposphere by NWP centres, but only now is it starting to be assimilated in the stratosphere. This is chiefly due to its important role in the radiation budget of the atmosphere, especially in the upper troposphere/lower stratosphere (UTLS) region, because it provides information on the atmospheric circulation, because it is a source of $\mathrm{HO}_{\mathrm{x}}\left(=\mathrm{OH}+\mathrm{HO}_{2}\right.$, involved in the catalytic destruction of ozone), and because it is a constituent of the Polar Stratospheric Clouds (PSCs) involved in polar ozone loss (Dessler, 2000).

In this section, we highlight some of the key issues concerning the assimilation of stratospheric water vapour. First, the stratosphere is very dry; while condensation of water vapour is commonplace in the troposphere, clouds (PSCs) only form in the stratosphere in the polar night, where extremely cold temperatures occur. Throughout the troposphere and the stratosphere, the water vapour mixing ratio varies by many orders of magnitude, from a few percent (by mass) in the tropical lower troposphere to a few parts per million (by mass or volume) in the stratosphere. In the stratosphere itself, the water vapour mixing ratio varies little, from $\sim 2$ parts per million by volume near the tropopause to $\sim 8$ parts per million by volume near the stratopause.

A second key issue is the available observations of water vapour. The primary source of moisture measurements is the radiosonde network. Radiosondes carry sensors that are primarily designed to measure the high relative humidity (RH) typical of the lower and middle troposphere. Where the humidity is low and temperature cold, as in the stratosphere, the measurements become less accurate (relatively, if not absolutely). Thus, routine radiosonde humidity measurements are of little or no use in the stratosphere, even if the sondes reach that level. More recently, satellite data have become more widely available, and are now used as an integral part of the operational assimilation of moisture information (e.g. ATOVS and SSM/I). However, the operational nadir soundings have relatively poor vertical resolution.

The large variation in humidity between the surface and the stratopause, together with different priorities in the troposphere (description of precipitation and identification of clouds) and the stratosphere (description of tracer distributions), means that it is difficult to specify a control variable suitable for use throughout the domain of models that span this region.

Dee and da Silva (2003) introduce a pseudo-relative humidity $\left(\mathrm{RH}^{*}\right)$, defined by scaling the mixing ratio $q$ by the saturation mixing ratio of the background field. An advantage of this approach is that a univariate $\mathrm{RH}^{*}$ analysis preserves $q$ in the absence of moisture observations. By contrast, using unmodified $\mathrm{RH}$ as a control variable implies a change in scaling if the temperature is changed, leading to changes in $q$ in the absence of moisture observations. In the presence of multivariate observations, this approach produces analysed humidity values that are close to those produced by a RH analysis.

In a parallel development, Hólm et al. (2002) introduced a normalized RH control variable, in which RH is divided by (an approximation of) the background variability. The new control variable has background errors that are more nearly Gaussian and homogeneous. Relating the normalization term of the new control variable to analysed $\mathrm{RH}$, as opposed to background $\mathrm{RH}$, also removes most of the skewness in the background errors seen for very dry or near-saturated air. Using normalized $\mathrm{RH}$, the assimilation scheme also takes better account of the large variability in the background error covariance matrix. This should improve the interpretation of 
Table 2. Summary of selected NWP- and GCM-based data assimilation experiments.

\begin{tabular}{|c|c|c|c|}
\hline References & Method & Assimilated constituent dataset & Chemistry \\
\hline $\begin{array}{l}\text { Caplan et al., 1997; Derber et al., 1998; } \\
\text { Derber and Wu, 1998; NCEP; this sys- } \\
\text { tem became operational in } 1997\end{array}$ & $\begin{array}{l}\text { Spectral statistical } \\
\text { interpolation }\end{array}$ & $\begin{array}{l}\text { Ozone a prognostic variable to improve } \\
\text { assimilation of radiances }\end{array}$ & None \\
\hline $\begin{array}{l}\text { Struthers et al., 2002; DARC/Met Of- } \\
\text { fice }\end{array}$ & Analysis correction & $\begin{array}{l}\text { Ozone profiles (UARS MLS); total col- } \\
\text { umn ozone (GOME) }\end{array}$ & Ozone parametrization; no cold tracer \\
\hline $\begin{array}{l}\text { Jackson and Saunders, 2002; Jackson, } \\
\text { 2004, 2007; Met Office }\end{array}$ & 3D-Var & $\begin{array}{l}\text { Ozone layers (SBUV/2); SBUV/2 and } \\
\text { ozone profiles (EOS MLS) in Jackson, } \\
2007\end{array}$ & $\begin{array}{l}\text { Ozone parametrization with cold tracer } \\
\text { (no cold tracer in Jackson and Saun- } \\
\text { ders, 2002; Jackson, 2007) }\end{array}$ \\
\hline $\begin{array}{l}\text { Dethof, 2003; ECMWF; this system } \\
\text { became operational in } 2002\end{array}$ & 4D-Var & $\begin{array}{l}\text { Ozone profiles (MIPAS); ozone lay- } \\
\text { ers (SBUV/2); total column ozone } \\
\text { (GOME) }\end{array}$ & $\begin{array}{l}\text { Ozone parametrization with heteroge- } \\
\text { neous chemistry term }\end{array}$ \\
\hline $\begin{array}{l}\text { Bormann et al., 2005, 2007; Bor- } \\
\text { mann and Healy, 2006; Bormann and } \\
\text { Thépaut, 2007; ECMWF }\end{array}$ & 4D-Var & $\begin{array}{l}\text { MIPAS limb infrared radiances sensi- } \\
\text { tive to ozone and humidity }\end{array}$ & $\begin{array}{l}\text { Ozone parametrization with heteroge- } \\
\text { neous chemistry term }\end{array}$ \\
\hline Dethof and Hólm, 2004; ECMWF & 3D-Var & $\begin{array}{l}\text { Ozone layers (SBUV/2); total column } \\
\text { ozone (TOMS) }\end{array}$ & $\begin{array}{l}\text { Ozone parametrization with heteroge- } \\
\text { neous chemistry term }\end{array}$ \\
\hline $\begin{array}{l}\text { Polavarapu et al., 2005a; Met Service } \\
\text { Canada }\end{array}$ & 3D-Var & $\begin{array}{l}\text { Simulated ozone tested within the as- } \\
\text { similation system }\end{array}$ & $\begin{array}{l}\text { Comprehensive stratospheric chem- } \\
\text { istry; gas-phase and heterogeneous re- } \\
\text { actions }\end{array}$ \\
\hline Lahoz et al., 2005; DARC/Met Office & 3D-Var & $\begin{array}{l}\text { Simulated ozone profiles (SWIFT, } \\
\text { IASI, GOME-2) }\end{array}$ & Ozone parametrization; no cold tracer \\
\hline Lahoz et al., 2007; DARC/Met Office & 3D-Var & Ozone and humidity profiles (MIPAS) & Ozone parametrization with cold tracer \\
\hline $\begin{array}{l}\text { Geer et al., 2006a, b, 2007; DARC/Met } \\
\text { Office }\end{array}$ & 3D-Var & $\begin{array}{l}\text { Ozone profiles (MIPAS); HIRS } 9 \text { (in } \\
\text { Geer et al., 2006b) }\end{array}$ & $\begin{array}{l}\text { Ozone parametrization with cold tracer } \\
\text { (no cold tracer in Geer et al., 2006b) }\end{array}$ \\
\hline
\end{tabular}

humidity data, and the mapping of information from radiances into temperature and humidity fields.

Further developments are currently under way at a number of NWP centres (e.g. ECMWF, Met Office), with the aim of developing an approach to moisture assimilation that performs well in both troposphere and stratosphere.

\subsection{Assimilation of ozone}

The main motivation for the inclusion of ozone data assimilation in operational NWP has been to take better account of ozone (in particular stratospheric ozone) when assimilating satellite radiance data, mainly from nadir sounding instruments. Radiance assimilation has been shown to improve the overall skill of weather forecasts (Saunders et al., 1999; McNally et al., 2006). Many of the channels used for atmospheric temperature sounding are at least partially sensitive to ozone, so improvements in the accuracy of ozone profiles can lead to more accurate temperature inversions.

At the same time, the assimilated ozone data can be used by the model radiation scheme, potentially leading to better radiative forcing of the model. Model radiation schemes take into account the absorption and emission of both shortwave (visible and near-UV) and long-wave (infrared) radiation by a number of atmospheric constituents. In the stratosphere, ozone is the dominant contributor to radiative heating, but the values are generally taken from ozone climatolo- gies (e.g. Fortuin and Kelder, 1998). An estimate of the true ozone distribution is likely to improve these calculations.

At ECMWF, ozone is already included in the forward modelling of satellite radiances. Experiments at ECMWF, using analysed ozone in heating rate calculations, found that variations in ozone amounts of $\sim 10 \%$ could result in changes in analysed UTLS temperatures of $2 \mathrm{~K}-4 \mathrm{~K}$ (Cariolle and Morcrette, 2006). Model runs with comprehensive chemistry and fully interactive ozone show significant temperature differences of up to $3 \mathrm{~K}$ in the upper stratosphere and lower mesosphere, compared with those with climatological ozone (Sassi et al., 2005). A prognostic ozone field allows the modelling of feedbacks between radiation, chemistry and dynamics, and this is expected to improve forecasts, especially over longer timescales. However, work by Morcrette (2003) suggests that coupling of the analysed ozone with the radiation scheme does not always bring improvement, and Cariolle and Morcrette (2006) state that in order to adequately represent the ozone radiative heating in the UTLS, ozone profiles with a vertical resolution of $\sim 1 \mathrm{~km}$ need to be assimilated. Recent experiments at the Met Office have shown that the inclusion of ozone-radiation feedbacks leads to an increase in the quality of tropospheric temperature, wind and geopotential height forecasts (Mathison et al., 2007). However, these changes are small and as yet not well understood, and the greatest impact of the ozone-radiation feedback is on analysed and forecast temperatures near the stratopause. 
An additional motivation for ozone assimilation is that the motion of ozone in the atmosphere could give useful dynamical information. Daley (1995) pointed out the feasibility of estimating the wind field from constituent observations, given sufficiently dense, frequent and accurate measurements. Riishøjgaard (1996) demonstrated the use of ozone measurements to reconstruct the flow field in a barotropic vorticity equation model. Peuch et al. (2000) demonstrated the dynamical impact of total ozone column observations in Observing System Simulation Experiments (OSSEs) using a 4D-Var data assimilation system. However, the use of ozone data to infer dynamical information is not without its problems. An inappropriately specified background error covariance matrix can lead to unrealistic impacts of ozone measurements on the wind fields. So, in practice, many ozone assimilation systems treat ozone as a univariate variable.

A further motivation for ozone assimilation is UV forecasting. Burrows et al. (1994) set up a system for operational UV forecasts in Canada. First, a field of total column ozone over the northern hemisphere is calculated using climatological total ozone column data, modified using regression relationships with a range of meteorological forecast fields (including vorticity, temperature and geopotential height) in the upper troposphere and stratosphere. Second, the total column ozone is corrected to fit ozone measurements over Canada. Finally, the clear-sky UV index is calculated using the solar zenith angle and day of the year. Other operational centres have developed similar systems (Austin et al., 1994, for the Met Office). An operational ozone DA system could be used to replace the first two steps of the procedure, with potentially better accuracy. The Australian Bureau of Meteorology already does something similar (Lemus-Deschamps et al., 2005), using a simplified analysis and forecast of TOVS total column ozone. This system, and that used at NCEP (Long, 2003) have the benefit of using a radiative transfer model to calculate the surface UV, rather than the empirical methods used in Canada and the UK.

In the stratosphere, ozone has a life-time ranging from $\sim 100$ days (lower stratosphere) to less than 1 day (upper stratosphere) (Dessler, 2000). Except in the upper stratosphere, these timescales for ozone are relatively long compared to the length of a typical weather forecast, which is of the order of days. So, in that context, the full treatment of chemical sources and sinks of ozone has not been a priority. Indeed, the use of a complex representation of ozone chemistry in an NWP system would be judged an unjustified overhead. Instead, the usual approach has been to implement simplified representations of ozone production and loss processes.

In early data assimilation systems, any representation of chemistry was omitted and ozone was treated as a passive tracer. Because ozone behaves as a passive tracer in the lower stratosphere (except under ozone hole conditions), this approach can provide useful information on the stratospheric ozone distribution (Polavarapu et al., 2005a). More recent developments have incorporated simple linear parametrizations of the chemical sources and sinks of ozone, typically known as Cariolle schemes (Cariolle and Déqué, 1986; McLinden et al., 2000; McCormack et al., 2004, 2006; Cariolle and Teyssèdre, 2007).

In the Cariolle scheme, the rate of change of ozone due to photochemistry $(C)$ is written as a first-order Taylor series expansion:

$C=a+b\left(\chi-\chi_{0}\right)+c\left(T-T_{0}\right)+d\left(\Phi-\Phi_{0}\right)$.

The first term in Eq. (3), $a$, is the equilibrium production minus loss, at the appropriate level and latitude. The second term accounts for differences between the current ozone amount $\chi$ and its equilibrium value, and the third for differences in the temperature $T$. The last term allows for solar radiation by considering the effect of the total ozone column $\Phi$ above the point under consideration. The coefficients $a$, $b, c$ and $d$ in Eq. (3), as well as the equilibrium values, are derived from a full chemistry model (usually a 2-D model), so the parametrized photochemistry is highly dependent on the particular model used. Geer et al. (2007) compare results from a range of linear chemistry ozone parametrizations and highlight some large differences.

The Cariolle schemes, contrary to some perceptions that they are non-rigorous, are actually based on sound photochemical arguments (see McCormack et al., 2006, for more details). Equation (3) springs directly from a linearized expansion of the fundamental odd-oxygen photochemical production and loss rate equations. This was done initially for pure oxygen (Chapman) photochemistry (Lindzen and Goody, 1965), and subsequently extended to reactions involving nitrogen, hydrogen and chlorine species (Blake and Lindzen, 1973; Stolarski and Douglass, 1985).

The scheme described by Eq. (3) does not take into account heterogeneous ozone chemistry, which is dominant under ozone hole conditions (Dessler, 2000). To remedy this shortcoming, the approach expressed in Eq. (3) can be modified to include a cold tracer to parametrize ozone loss due to heterogeneous processes (Hadjinicolau et al., 1997; Eskes et al., 2003). The cold tracer approach is not the only means by which heterogeneous ozone loss is represented in ozone data assimilation. Cariolle and Teyssèdre (2007) describe a version of the Cariolle scheme that represents this ozone loss without using a cold tracer, and ECMWF uses a version with this approach, too (Dethof, 2003).

The relaxation rate $\tau=-1 / b$ corresponds directly to the ozone photochemical lifetime. As shown by Geer et al. (2006a, 2007), the values of $\tau$ confirm that in the lower stratosphere ( $\tau \sim 100$ days) the photochemistry could be neglected, but in the upper stratosphere ( $\tau \sim 0.5$ days) the photochemistry is very important. But, it follows that, if the photochemical coefficients and equilibrium values are not realistic, the ozone data will quickly relax to an incorrect value, ignoring information from observations. In such circumstances, the parametrized chemistry scheme will seriously degrade 
the assimilated ozone fields in the upper stratosphere, and it may be preferable to omit the chemistry.

Results reported in the ASSET analysis intercomparison project (Geer et al., 2006a) where ozone analyses from several GCMs and CTMs are compared for a fixed time period, show that, for current ozone data assimilation systems, with good ozone observations and no chemistry one can get a good representation of the ozone field even when the photochemistry timescales are fast. However, above $0.5 \mathrm{hPa}$, where the ozone diurnal cycle is no longer negligible, only analyses with a detailed representation of mesospheric chemistry capture it. Finally, provided that there are no observational gaps, the complexity of the chemical scheme tends to have little effect on the quality of the ozone analyses. However, these results also show that observational gaps can seriously degrade the ozone analyses. Arguably, in the upper stratosphere (fast chemical time-scales), a better solution than omitting chemistry would be to bias correct the Cariolle scheme (see, e.g., Coy et al., 2007).

The first implementation of an ozone assimilation system for operational NWP was at the NCEP (Caplan et al., 1997; Derber et al., 1998). A univariate ozone assimilation was included in the operational ECMWF 4D-Var system in April 2002, and was also part of the 3D-Var system for the ERA-40 re-analysis (Dethof and Hólm, 2004; Uppala et al., 2005). ECMWF also currently provide analyses and forecasts of ozone (Dethof, 2003). Of necessity, ozone assimilation systems for NWP are limited to using measurements that are available close to real time. This effectively means data from SBUV/2 (retrievals) and HIRS (channel 9 radiances), both carried by the NOAA polar-orbiter satellites. However, ozone data from research satellites can also be available in close to real time: ECMWF have assimilated operationally ozone profile data from MIPAS and total column ozone data from GOME, and at the time of writing (October 2007) are assimilating total column ozone data from SCIAMACHY (R. Dragani, personal communication, 2007). TOMS total column ozone data have been assimilated for re-analyses (Dethof and Hólm, 2004). Some of these satellite instruments give only restricted vertical coverage; for example, HIRS channel 9 is most sensitive to the lower-stratosphere ozone maximum, while SBUV/2 retrievals give some profile information above the ozone peak in the mid stratosphere.

Ozone assimilation has also been developed at the Met Office, first using the analysis correction scheme (Connew, 1999; Struthers et al., 2002), and later 3D-Var (Jackson, 2004, 2007; Geer et al., 2006b) - see Fig. 1. Other NWP centres, e.g., GMAO and the Royal Netherlands Meteorological Institute (KNMI) have taken the approach of developing an ozone analysis in a CTM driven by assimilated wind and temperature data (see Sect. 4).

For non-operational systems (and, increasingly, operational systems such as that of ECMWF) that assimilate research satellite data from platforms such as ESA's Envisat, the situation is better than with traditional operational satel- (a)

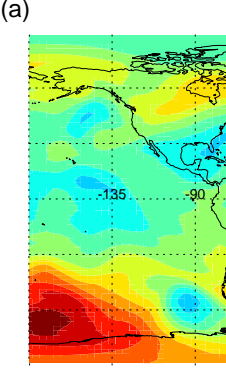

(b)

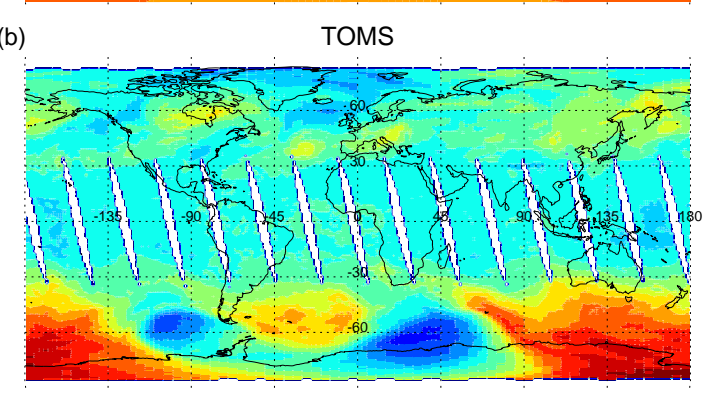

(c)

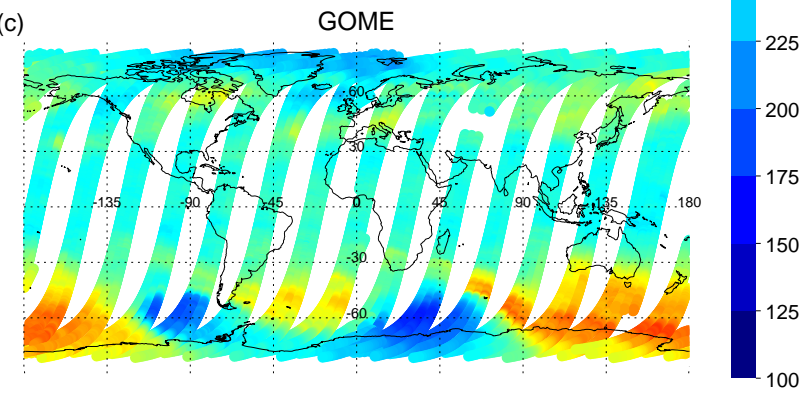

Fig. 1. Total column ozone on 26 September 2002 (Dobson Units, DU) from (a) the 12:00 UT troposphere-stratosphere Met Office analysis with the column ozone below $200 \mathrm{hPa}$ replaced by an ozone climatology; (b) TOMS; (c) GOME. Based on Geer et al. (2006b). (c) Royal Meteorological Society.

lite data (e.g. SBUV/2, HIRS channel 9 radiances). In this case both nadir and limb sounders are used, with the latter providing better vertical resolution because of their viewing geometry. There is recent evidence that adding heightresolved ozone data improves ozone analyses in an NWP system. In the intercomparison of ozone analyses described by Geer et al. (2006a), it is shown that assimilation of heightresolved MIPAS ozone data improves the ECMWF NWP ozone analyses. This improvement is attributed to the benefit coming from the relatively high vertical resolution of MIPAS, and the fact that before this only limited ozone data were assimilated (namely, SBUV/2 ozone layers and GOME total column ozone). A similar improvement is seen in the Met Office system, where assimilation of height-resolved EOS MLS ozone data reduces analyses errors compared to the situation when only SBUV/2 ozone layers are assimilated (Jackson, 2007). These results suggest a way forward toward improved use of ozone data in NWP systems. Along these lines, benefit could be expected from the assimilation of 
height-resolved ozone data from the Metop IASI instrument, and from the AIRS instrument on the EOS Aqua platform (see Table 1).

While ozone assimilation systems have focused almost exclusively on satellite data, it would also be possible to use ground based ozone measurements. The main reasons why they are not generally used is first their scarcity and second that they have not been routinely exchanged alongside other meteorological data. Ozonesondes are expensive to make - much more expensive than radiosondes, themselves under economic pressure. As a result, ozonesondes tend to be flown routinely once a week from a very limited number of stations, plus during certain research campaigns, such as MATCH (Streibel et al., 2006). While the scarcity of groundbased ozone data means that it is not worthwhile assimilating them routinely, they are a very valuable data set for the validation of ozone assimilation systems. There are a larger number of Dobson measurements of total column ozone, but these have no profile information, as well as being sparse compared to satellite measurements.

\section{Chemical model approaches}

For constituent assimilation, there are several good reasons for avoiding the use of NWP models, and instead using what we refer to as the chemical model approach. First, NWP models are complex and generally expensive in terms of computer resources. Second, they tend to focus on the dynamics of the atmosphere, so that, typically, only constituents that interact with the dynamics are represented. This is the case for ozone and water vapour (see Sect. 3). In NWP models, chemistry is commonly parametrized to simplify the system, so that in some cases (to be discussed later) this set-up can be inappropriate.

If the goal is not to improve the weather forecast, other types of model are more appropriate for constituent assimilation. In particular, (i) photochemical box models along an air parcel trajectory, and (ii) three dimensional CTMs. In both these cases, the dynamical problem is simplified because the dynamical fields are pre-calculated from a NWPbased system. In the first case, the trajectory and the atmospheric conditions (temperature, pressure) along it are given and a photochemical box model is used to calculate the evolution of the composition in the transported air parcel. In the second case, wind and temperature fields are prescribed and used to advect the constituents in the model. The chemical scheme used by CTMs varies in complexity and depends on the final application. If the assimilation system focuses on long-lived species (chemistry timescales $\gg$ transport timescales), e.g. methane and $\mathrm{N}_{2} \mathrm{O}$ in the lower stratosphere, chemistry can generally be neglected. If the assimilation system focuses on ozone, where both chemistry and transport can be important in the stratosphere, a parametrized chemical scheme can be sufficient. If the assimilation sys- tem focuses on reactive, i.e., short-lived species (chemistry timescales « transport timescales), e.g. $\mathrm{NO}_{2}$ in the stratosphere, then explicit calculation of the chemical interactions is generally necessary. The first two cases are cheaper in computer time than the third one. The cost of computer time is another important factor to consider in constituent assimilation.

In general, there is more variability in the data assimilation set-up of chemical model systems than in that for NWP model systems. This is also reflected in the number of applications of the former. Currently, chemical model assimilation systems are used to: (i) derive information on unobserved species (Errera and Fonteyn, 2001; Lary et al., 2003); (ii) test chemical theories (Lary et al., 2003; Marchand et al., 2003); (iii) design constituent measurement strategies (Khattatov et al., 2001); (iv) provide analyses of tropospheric pollution (Elbern et al., 2007); (v) support the evaluation of satellite instruments (Marchand et al., 2004; Vigouroux et al., 2007); (vi) monitor stratospheric ozone (Levelt et al., 1998; El Serafy et al., 2002; Eskes et al., 2003; Štajner et al., 2004; Štajner and Wargan, 2004; Massart et al., 2004; Segers et al., 2005; Wargan et al., 2005; Rösevall et al., 2007a, b); (vii) monitor stratospheric chemical species other than ozone, e.g., $\mathrm{NO}_{2}, \mathrm{CH}_{4}, \mathrm{~N}_{2} \mathrm{O}$, and aerosol (Khattatov et al., 2000; Ménard et al., 2000; Ménard and Chang, 2000; Collins et al., 2001; Errera and Fonteyn, 2001; Fonteyn et al., 2001; Chipperfield et al., 2002; El Amraoui et al., 2004); and (viii) forecast stratospheric ozone: at KNMI (Eskes et al., 2002, 2005; El Serafy and Kelder, 2003), at the GMAO (Riishøjgaard et al., 2000; Štajner et al., 2001), and at the Belgian Institute for Space Aeronomy, BIRA-IASB (http:// www.bascoe.oma.be/). BIRA-IASB also have provided forecasts of PSC surface area density, $\mathrm{ClO}_{\mathrm{x}}\left(=\mathrm{ClO}+2 * \mathrm{Cl}_{2} \mathrm{O}_{2}\right)$, $\mathrm{N}_{2} \mathrm{O}, \mathrm{HNO}_{3}$ and $\mathrm{ClONO}_{2}$. Recent reviews of data assimilation using chemical models include those by Lary (1999), Wang et al. (2001) and Khattatov (2003). Table 3 provides a summary of selected chemical model data assimilation experiments.

To attain the broader goals of data assimilation using chemical models, several data assimilation methods are used: successive correction; optimal interpolation (OI), the KF and variants thereof; variational methods (3D- and 4D-Var); and PSAS (3D-PSAS, the dual of 3D-Var, is, to our knowledge, the only form of PSAS to have been used so far on stratospheric constituent assimilation). By contrast, most current NWP systems are based on variational methods.

In the following part of this section, we review the different methods and systems used in constituent data assimilation with chemical models. We will also point out the major differences between these systems and the systems based on NWP models. For example, CTM-based systems tend to not consider radiance assimilation, which is generally the case in operational NWP systems (This is not due to a fundamental limitation of CTMs, which can theoretically be used with complicated observation operators - see, e.g., Müller et al., 
Table 3. Summary of selected chemical model data assimilation experiments.

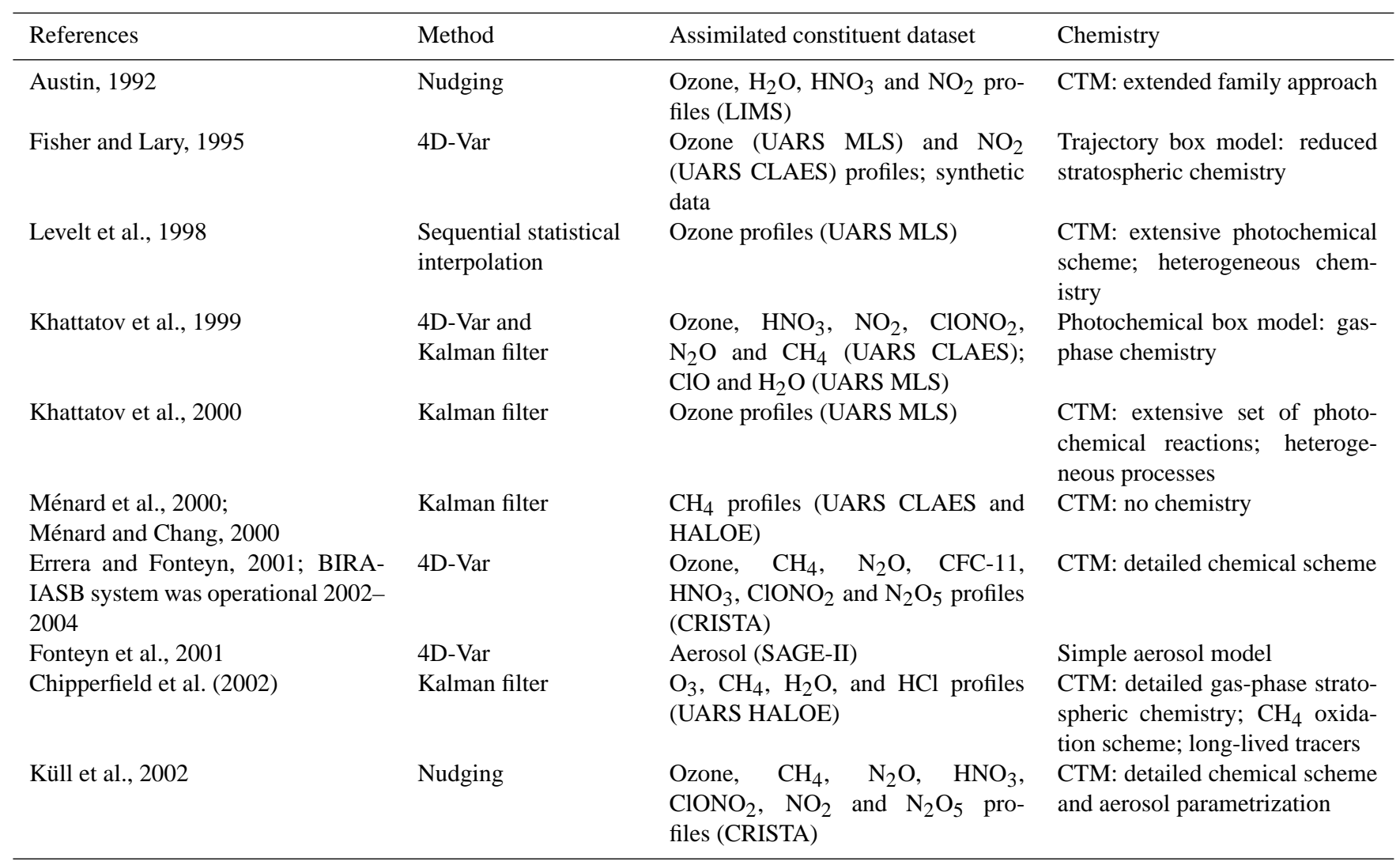

2004.). For CTM-based systems, the observations are previously inverted to provide profiles or total column. In the case of profiles, the observation operator is reduced to the spatial interpolation of the model values at the observation location. In the case of columns, the model values are integrated over the model layers before performing the spatial interpolation. A second important point concerns the case where CTMs use a full photochemical scheme. In this case, the number of constituent control variables is much greater than in an NWP system. To give an example, a modern stratospheric CTM includes $\sim 50$ chemical species while the current ECMWF NWP system includes only two constituents (humidity and ozone).

Three methods are commonly used in constituent data assimilation with chemical models (see Sect. 2.2 for an introduction): 4D-Var, approximations to the Kalman Filter (generally involving parametrizations of the error covariances), and PSAS (which can be viewed as an approach to solve the Kalman filter, or as the dual of 3D-Var). Each of these methods has advantages and disadvantages. The feasibility of 4D-Var has been demonstrated in NWP systems. Its main advantage is that it considers observations over a time window that is generally much longer than the model time step: typically $24 \mathrm{~h}$ for chemical models, while the CTM time step is of the order of $30 \mathrm{~min}$ or less. This allows more observations to constrain the system and, considering satellite coverage, increases the geographical area influenced by the data. For non-linear systems (as is generally the case for the atmosphere), this feature of 4D-Var, together with the nondiagonal nature of the adjoint operator which transfers information from observed regions to unobserved regions, reduces the weight of the background error covariance matrix in the final 4D-Var analysis compared to the KF analysis (for linear systems, the general equivalence between 4D-Var and the KF implies that the same weight is given to all data in both systems). In the case of constituent assimilation where a full photochemistry scheme is considered, the properties of the adjoint operator allow unobserved species to be constrained by observed species. This constraint can be expected when observed and unobserved species chemically interact with a time scale of the order of the assimilation window or less. A special property of the 4D-Var analysis is that in the middle of the assimilation window it uses all of the observations simultaneously, not just those before the analysis. Because of this, 4D-Var is said to be a smoothing algorithm. 
Table 3. Continued.

\begin{tabular}{|c|c|c|c|}
\hline $\begin{array}{l}\text { Eskes et al., 2002, 2003, 2005; } \\
\text { Segers et al., 2005; KNMI system } \\
\text { became operational in } 2000\end{array}$ & Kalman filter & $\begin{array}{l}\text { Total column ozone (GOME); } \\
\text { ozone profiles (GOME) in Segers et } \\
\text { al. }\end{array}$ & $\begin{array}{l}\text { CTM: ozone parametrization; } \\
\text { cold tracer }\end{array}$ \\
\hline Lary et al., 2003 & Kalman filter & $\begin{array}{l}\text { Ozone, } \mathrm{NO}, \mathrm{NO}_{2}, \mathrm{~N}_{2} \mathrm{O}_{5}, \mathrm{HNO}_{3} \text {, } \\
\mathrm{HO}_{2} \mathrm{NO}_{2}, \mathrm{HCN}, \mathrm{ClONO}_{2}, \mathrm{HCl} \text {, } \\
\mathrm{H}_{2} \mathrm{O}, \mathrm{CO}, \mathrm{CO}_{2}, \mathrm{CH}_{4} \text {, and } \mathrm{N}_{2} \mathrm{O} \text { pro- } \\
\text { files (ATMOS) }\end{array}$ & $\begin{array}{l}\text { Stacked photochemical box } \\
\text { models: comprehensive chem- } \\
\text { istry }\end{array}$ \\
\hline Marchand et al., 2003, 2004 & $\begin{array}{l}\text { 4D-Var on a } \\
\text { box model }\end{array}$ & $\begin{array}{l}\text { Ozone, } \mathrm{NO}_{2}, \mathrm{NO}_{3} \text { profiles }(\mathrm{GO}- \\
\text { MOS) }\end{array}$ & $\begin{array}{l}\text { Photochemical box model: gas- } \\
\text { phase chemistry; hetereogenous } \\
\text { processes }\end{array}$ \\
\hline $\begin{array}{l}\text { Štajner et al., 2001, 2004, 2006; } \\
\text { Stajner and Wargan, 2004; Wargan } \\
\text { et al., 2005; GMAO system became } \\
\text { operational in } 1999\end{array}$ & PSAS & $\begin{array}{l}\text { Total column ozone (TOMS); ozone } \\
\text { layers (SBUV/2); ozone profiles } \\
\text { (POAM-III). A prototype for assim- } \\
\text { ilating SBUV/2 radiances has been } \\
\text { tested (Müller et al., 2004) }\end{array}$ & $\begin{array}{l}\text { CTM: ozone parametrization; } \\
\text { no chemistry (Štajner et al., } \\
\text { 2001); ozone transport imple- } \\
\text { mented with the GEOS-4 GCM } \\
\text { (Štajner et al., 2006) }\end{array}$ \\
\hline El Amraoui et al., 2004 & $\begin{array}{l}\text { Sequential statistical } \\
\text { interpolation }\end{array}$ & Ozone, $\mathrm{N}_{2} \mathrm{O}$ profiles (ODIN SMR) & $\begin{array}{l}\text { CTM: comprehensive gas-phase } \\
\text { chemistry and heterogeneous re- } \\
\text { actions }\end{array}$ \\
\hline Massart et al., 2004 & 3D-FGAT & Ozone profiles (GOME) & CTM: detailed photochemistry \\
\hline Baier et al., 2005 & OI & $\begin{array}{l}\text { Ozone, } \mathrm{H}_{2} \mathrm{O}, \mathrm{NO}_{2}, \mathrm{CH}_{4}, \mathrm{~N}_{2} \mathrm{O} \text { and } \\
\mathrm{HCl} \text { profiles (MIPAS) }\end{array}$ & $\begin{array}{l}\text { CTM: detailed gas phase chem- } \\
\text { istry and heterogeneous pro- } \\
\text { cesses on sulphuric acid aerosols }\end{array}$ \\
\hline Coy et al., 2007 & PSAS & Ozone layers (SBUV/2) & $\begin{array}{l}\text { CTM data assimilation scheme } \\
\text { (Štajner et al., 2006) coupled to } \\
\text { a GCM: ozone parametrization } \\
\text { (some experiments do not have } \\
\text { chemistry). The Coy et al. study } \\
\text { suggests this set-up fits some- } \\
\text { where between the NWP- and } \\
\text { CTM-based approaches; it was } \\
\text { a first step toward developing a } \\
\text { full NWP-based approach using } \\
\text { 3D-Var (S. Eckermann, personal } \\
\text { communication, 2007) }\end{array}$ \\
\hline Rösevall et al., 2007a, b & Kalman filter & $\begin{array}{l}\text { Ozone profiles (ODIN/SMR) in } \\
\text { Rösevall et al. (2007a); ozone } \\
\text { profiles (MIPAS, ODIN/SMR) in } \\
\text { Rösevall et al. (2007b) }\end{array}$ & CTM: no chemistry \\
\hline
\end{tabular}

In contrast with the above advantages of 4D-Var, three weaknesses must be mentioned. First, its numerical cost is very high compared to approximate versions of the KF, and to 3D-PSAS, so that, in general, its implementation requires a supercomputer. The cost of $4 D-P S A S$ (the dual of 4DVar), like the cost of 4D-Var, is determined by the cost of the repeated integrations of the assimilating model and its adjoint (see, e.g., Courtier, 1997; Louvel, 2001); thus, its cost (if implemented for stratospheric constituent data assimilation) would not be significantly lower compared to that of 4D-Var. Second, its formalism cannot determine the analysis error directly; rather it has to be computed from the inverse of the Hessian matrix (again, this procedure is prohibitive in both CPU and memory). Finally, in contrast with NWP 4D-Var systems, past assimilation experiments using CTMs have not been based on the incremental method (Bouttier and Courtier, 1999) and thus cannot take advantage of its benefits, e.g., solving the analysis at a reduced resolution, thereby reducing the computational cost.

The first assimilation study of constituent observations based on 4D-Var was presented by Fisher and Lary (1995). They used a trajectory box model with a reduced stratospheric chemistry scheme involving $\mathrm{O}_{3}, \mathrm{O}, \mathrm{NO}, \mathrm{NO}_{2}$ and $\mathrm{N}_{2} \mathrm{O}_{5}$. They assimilated $\mathrm{O}_{3}$ and $\mathrm{NO}_{2}$ data from the MLS and CLAES instruments on board NASA's Upper Atmosphere Research Satellite (UARS). They also performed an 
assimilation experiment using synthetic, i.e., simulated, data that showed ozone observations were able to constrain the other species. This study also introduced the concept of the influence function which, with the help of the adjoint model, measures the influence of an observed species at time $t>t_{0}$ on other modelled species at the initial time, $t_{0}$.

Errera and Fonteyn (2001) built a 4D-Var assimilation system for stratospheric chemical observations. This system is based on a three-dimensional CTM with a detailed chemical scheme including 41 species and 144 reactions. Observations are taken from the CRISTA instrument. These include long-lived species $\left(\mathrm{CH}_{4}, \mathrm{~N}_{2} \mathrm{O}\right.$ and $\left.\mathrm{CFC}-11\right)$ and species with relatively shorter lifetimes $\left(\mathrm{O}_{3}, \mathrm{HNO}_{3}, \mathrm{ClONO}_{2}\right.$ and $\left.\mathrm{N}_{2} \mathrm{O}_{5}\right)$ in comparison to the time-scale of the assimilation window $(24 \mathrm{~h})$. Comparison with independent observations shows good agreement for observed species (e.g. 7\% for ozone against $\mathrm{HALOE}$; less than $15 \%$ for $\mathrm{HNO}_{3}$ against ATMOS), and for $\mathrm{NO}_{\mathrm{x}}\left(=\mathrm{NO}+\mathrm{NO}_{2}\right)$ and $\mathrm{HCl}$, two constituents that are not observed by CRISTA (in both cases less than $25 \%$ against HALOE). It was also shown that the $\mathrm{HCl}$ field is influenced by the assimilation of $\mathrm{ClONO}_{2}$ observations.

Because of the strong temperature-dependence of the chemistry of short-lived species such as $\mathrm{NO}_{2}$ and $\mathrm{NO}_{3}$, their variability could provide information on temperature. One possible application is the use of temperature as a control variable in a chemical DA system. Along these lines, the variational system built by Marchand et al. $(2003,2004)$ has been used to extract temperature information from GOMOS $\mathrm{NO}_{3}$ observations (Lahoz et al., 2007).

The two other methods commonly used for constituent data assimilation are approximate versions of the KF, and PSAS. The KF method is formulated so that the analyses uncertainties are determined directly and can be propagated to the next assimilation time step. The PSAS set-up at the GMAO includes a method to compute an approximation of the forecast error covariance matrix.

Approximate versions of the KF, and PSAS, are based on the hypothesis of model linearity. Thus, the time window over which observations can be considered should be chosen carefully to ensure that the linearity hypothesis is satisfied. Khattatov et al. (1999) provided evidence that for a stratospheric photochemical box model, the linear approximation essential to applicability of the EKF and 4D-Var is valid up to $\sim 10$ days. This behaviour is explained as the combination of two factors: (i) concentrations of many modelled shortlived constituents are largely determined by concentrations of a few relatively long-lived constituents such as ozone, and parameters such as total active chlorine or nitrogen; and (ii) within the data assimilation set-up, linear approximations are generated at every solver time step and the matrices corresponding to such linear transformations are multiplied to obtain a matrix approximating the evolution of the system over a 10-day period. Due to the nature of the stiff solvers, these time steps vary by orders of magnitude and get very small when the changes in concentration for some species are most rapid.

Lyster et al. (1997) developed a Kalman filter system for a two-dimensional advection model on an isentropic surface. Although particular effort was made to optimize the CPU time, such a system was not found to be practical due to the large computer resources required. Ménard et al. (2000), using the same model as Lyster et al. (1997) for the assimilation of $\mathrm{CH}_{4}$ data, found that the standard $\mathrm{KF}$ formalism propagated the analysis covariance matrix inaccurately, with rapid loss of variance and an increase in the error correlations. To remedy this shortcoming, they formulated an alternative formalism to the KF system. This alternative formalism, described in companion papers by Ménard et al. (2000) and Ménard and Chang (2000), estimates model parameters using a robust method based on $\chi^{2}$ diagnostics which compares the observation minus forecast $(\mathrm{OmF})$ residuals with those calculated by the Kalman filter (see also Sect. 5). The method is used to estimate three covariance parameters (representativeness error, model error, and initial error). Because correlation length-scale parameters are found to be insensitive to the $\chi^{2}$ diagnostics, they are estimated using a maximumlikelihood method. The $\chi^{2}$ diagnostics have been used in other studies to estimate data assimilation system parameters; statistics from the OmF time series are also used to estimate these parameters.

Khattatov et al. (2000) used the $\chi^{2}$ diagnostics with a three-dimensional CTM that assimilated ozone data. The multi-dimensional nature of the problem meant that some simplification was required to comply with limitations in computer resources, both in terms of CPU and memory. Khattatov et al. (2000) also showed that the value of $\chi^{2}$ primarily depends on the value of the error growth and not on the correlation distance. The same authors also found that the root-mean-square of the OmF differences is mainly sensitive to the correlation length in the case where the spatial density of observations is high.

The $\chi^{2}$ diagnostics methodology has been applied successfully in stratospheric data assimilation (e.g. Chipperfield et al., 2002; Fierli et al., 2002; Lary et al., 2003 and, with some modifications, by El Amraoui et al., 2004 and Baier et al., 2005). Chipperfield et al. (2002) also introduced a method to constrain unobserved long-lived species (e.g. $\mathrm{N}_{2} \mathrm{O}$ ), in which an observed long-lived species (e.g. $\mathrm{CH}_{4}$ ) is used to preserve a compact tracer-tracer relationship between both constituents. Finally, Eskes et al. (2003) developed a KF approach to produce near-realtime ozone analyses and five-day forecasts. To comply with limited computer resources and the constraints of an operational service, Eskes et al. (2003) introduced several approximations in the KF method. For example, they used observation minus forecast $(\mathrm{OmF})$ statistics to estimate the horizontal error correlations, the observation errors and the forecast errors. 
As can be seen from the above examples, approximate versions of Kalman filter methods are very popular for constituent assimilation. This popularity is due to their low demand for computer resources in comparison to 4D-Var, as well as no requirements for an adjoint model. An alternative to approximate versions of the KF is the PSAS method used at the GMAO. It has the advantage that it solves the analysis in the observation space, which, for constituent assimilation, is typically much smaller in size than the model space. It thus reduces the computer resources needed. This approach is used by the Goddard Earth Observation System (GEOS) ozone data assimilation system (Štajner et al., 2001). This system, based on a three-dimensional CTM with parametrized ozone chemistry, also uses the $\chi^{2}$ diagnostics to estimate the system parameters. The system has been operational since 1999, providing stratospheric ozone analyses using SBUV/2 and TOMS (Štajner et al., 2001). Other combinations of ozone datasets have been assimilated in experimental versions of the GMAO system: SBUV/2 and POAM-III (Štajner and Wargan, 2004), SBUV/2 and MIPAS (Wargan et al., 2005); and SBUV/2, POAM-III and ILAS-II (Štajner et al., 2006).

Finally, as well as considering the performance of the NWP-based and chemical model approaches, one also needs to address the relative costs. While cost differences depend on the complexity of different model components, one can still highlight some key factors.

First, it is significantly cheaper to use a transport model than a coupled chemistry/dynamics model, if dynamical fields are available already. In a test with the Met Office Unified Model, the dynamics took 25\% of the total model time, while advection of three tracers took 6\% (A. Malcolm, personal communication, 2005). The advection of a single tracer is relatively simple and cheap compared with the sophistication required by the dynamics of the Met Office model. Similarly, the cost of the univariate assimilation of a single constituent will be simpler and cheaper than the proportionate cost of a dynamical variable that is treated multivariately. Furthermore, the smaller data volume of constituent observations makes constituent data assimilation relatively cheaper than data assimilation of dynamical variables (e.g. temperature, winds, humidity).

On the other hand, costs of the constituent DA include the cost of the required chemistry model. While this could be simple (or even non-existent for long-lived constituents such as methane in the lower stratosphere), a complex chemical model is likely to be a major component of a sophisticated chemical DA system. While we have outlined a range of cost considerations, it is worth stressing that the costs are highly dependent on the type of DA method, transport model, and chemistry employed.

\section{Evaluation of models, observations and analyses}

Both NWP-based and chemical model data assimilation approaches (see Sects. 3 and 4) are used to evaluate models and observations, in particular concerning ozone (e.g. Štajner et al., 2004; Geer et al., 2006a, b, 2007; Coy et al., 2007). Data assimilation not only corrects weaknesses in models, but also identifies model deficiencies such as biases (e.g. between model and observations; between different observations), which as Rood (2005) states is likely the greatest current challenge in data assimilation. In this Sect. we provide further details.

A crucial element of data assimilation is the evaluation of the quality of the observations, the model and the analyses, and the test of several assumptions built into data assimilation algorithms, e.g., Gaussian errors; unbiased observations and models. Several diagnostics have been developed to do this (Talagrand, 2003c). Broadly speaking, these consist of: self-consistency tests, and independent tests. We first discuss self-consistency and independent tests in general. We then provide illustrative examples of how constituent data assimilation can be used to evaluate satellite instruments.

\subsection{Self-consistency tests}

Self-consistency tests provide useful information for evaluating the quality of the data assimilation ingredients and the assumptions built into assimilation algorithms. Histograms of OmA (observation minus analysis) and OmF (observation minus forecast) differences are computed for a range of spatial and temporal scales to test whether the observations, forecast and analysis fields, and their errors, are consistent with each other. For example, the OmA histogram should be more peaked than that for $\mathrm{OmF}$, as the analyses should be closer to the assimilated observations than the forecast. Furthermore, the OmF histogram should be Gaussian, if both the observation and forecast are assumed to have Gaussian errors. Time averages of the standard deviation of OmA can also be used to test whether the assimilation system is consistent with the concept of the Best Linear Unbiased Estimate, BLUE (Talagrand, 2003a), introduced in Sect. 2.1. Other tests check whether there are biases between observation and forecast, or between observation and analysis. Application of these tests is discussed in Errera and Fonteyn (2001), Štajner et al. (2001), Struthers et al. (2002) and Segers et al. (2005). See Fig. 2 for an example. Tests for Gaussian errors can also include tests of skewness and kurtosis (Geer et al. 2006b).

Time series of OmA and OmF differences test whether the observation, forecast and analysis fields, and their errors, are consistent with each other. A well-behaved data assimilation system will have time series with mean OmA and OmF values that are close to zero and do not vary much over time. If this is not true, a bias between the model and the data (or a subset of the data) is present. Also, if the standard deviation about the mean of the OmA time series is larger 
than the observational error, this indicates that the system is not properly set up. For example, the observation and background error covariance matrices, $\mathbf{R}$ and $\mathbf{B}$, respectively, could be poorly characterized. Desroziers et al. (2005) suggest a simple method to evaluate $\mathbf{R}$ and $\mathbf{B}$ separately; Chapnik et al. (2006) describe a way of quantifying errors and biases of both model and observations in the process of tuning a DA scheme for internal consistency.

Time series of OmA and OmF differences can also be used to monitor the performance of satellite instruments; changes in their values can indicate a change in the instrument algorithm, or a degradation of the instrument. For example, Štajner et al. (2004) uses the OmF time series provided by the GEOS ozone data assimilation system to validate the NOAA14 SBUV/2 retrieval algorithm. Furthermore, at the start of a data assimilation experiment, it can take some time for the system to spin-up; this spin-up time is shown by the time it takes for OmA or OmF differences to converge towards a constant value (Struthers et al., 2002).

Without chemistry it is difficult to identify instrument drift using OmF and OmA differences, as drifts in the observations are incorporated into the forecasts and analyses, and the differences do not change much as both forecasts and analyses incorporate the instrument drift. With chemistry, however, this can be remedied as the model pulls the biased observations toward a more typical state (e.g. the equilibrium reference state in Eq. (3) for ozone).

Regardless of the inclusion of chemisty, instrument drifts can be identified if at the same time as the assimilation is performed, the system is monitored with independent data. This instrument drift could be monitored with a free-running model, but in this case it would be difficult to attribute the source of the drift.

If the OmF differences have a Gaussian distribution, its inner product normalized by its covariance is a random variable that has a $\chi^{2}$ distribution with $p$ degrees of freedom, where $p$ is the number of observations. This result can be used to test whether the OmF differences are consistent with assumptions made in the assimilation algorithm, and to monitor the observations (Ménard et al., 2000; Ménard and Chang, 2000; Štajner et al., 2004).

If the data (observation and background) errors are Gaussian, the minimum of the penalty function, $J_{\min }$, follows a $\chi^{2}$ distribution with $p$ degrees of freedom, and must be equal on average to $p / 2$. This last result is also true if the errors are not Gaussian, but the assimilation scheme remains linear. Thus, in these cases, $J_{\min } / p$ should on average be 0.5 (Talagrand, $2003 \mathrm{c}$ ). In practice, $J_{\min } / p$ is often significantly different from 0.5 . This discrepancy can arise from an incorrect estimate of $\mathbf{B}$ or $\mathbf{R}$ (mainly the representativeness error in the case of $\mathbf{R}$ ).

Several robust correlations between pairs of long-lived tracers have been observed in the atmosphere (Plumb and Ko, 1992). A particular example is the correlation between $\mathrm{CH}_{4}$ and $\mathrm{N}_{2} \mathrm{O}$ (Chipperfield et al., 2002). When two or more
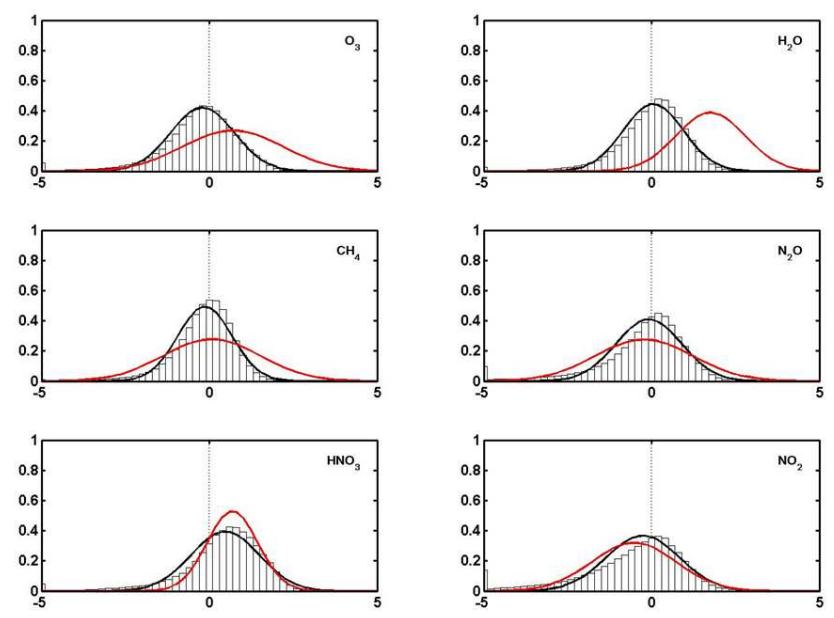

Fig. 2. Evaluation of analyses using histograms of OmF differences (normalized by the observation error) averaged for the stratosphere, the globe and August 2003 for six stratospheric constituents: $\mathrm{O}_{3}$ (top left), $\mathrm{H}_{2} \mathrm{O}$ (top right), $\mathrm{CH}_{4}$ (middle left), $\mathrm{N}_{2} \mathrm{O}$ (middle right), $\mathrm{HNO}_{3}$ (bottom left) and $\mathrm{NO}_{2}$ (bottom right). The constituent observations are from ESA MIPAS off-line retrievals. The frequency of the histograms is normalized by the observations, so that the sum of the histogram values is 1 . The black line is a Gaussian fit to the histograms; the red line is a Gaussian fit from a model run without assimilation. The results support the assumption of Gaussian errors in the observations and the forecast, and show the analyses are closer to the observations than simulations from the model run without assimilation. The experiments were performed at BIRA-IASB (http://www.bascoe.oma.be).

long-lived tracers are assimilated, the quality of the analyses can be assessed through the consistency of the tracer-tracer correlations.

\subsection{Independent tests}

These tests involve comparison of analyses with data that are independent from the analyses, i.e., data not assimilated to provide the analyses. Independent datasets used to evaluate ozone analyses include ozonesondes (Logan, 1999) or satellite data which are not commonly assimilated (e.g. the UARS HALOE instrument, Russell et al., 1993). Independent data can provide information on whether the analyses are realistic and can help attribute biases to observations, forecast and analysis; note that self-consistency tests cannot be used to perform this attribution. Estimating the bias in the analyses by comparison against independent data is only possible when the error characteristics of the latter are well known. Application of these tests is discussed in Khattatov et al. (2000), Struthers et al. (2002) and Segers et al. (2005). See Fig. 3 for an example.

When analyses are compared against independent data it is important to take account of the observation characteristics of each dataset. This can be accomplished by making use of 
(a)
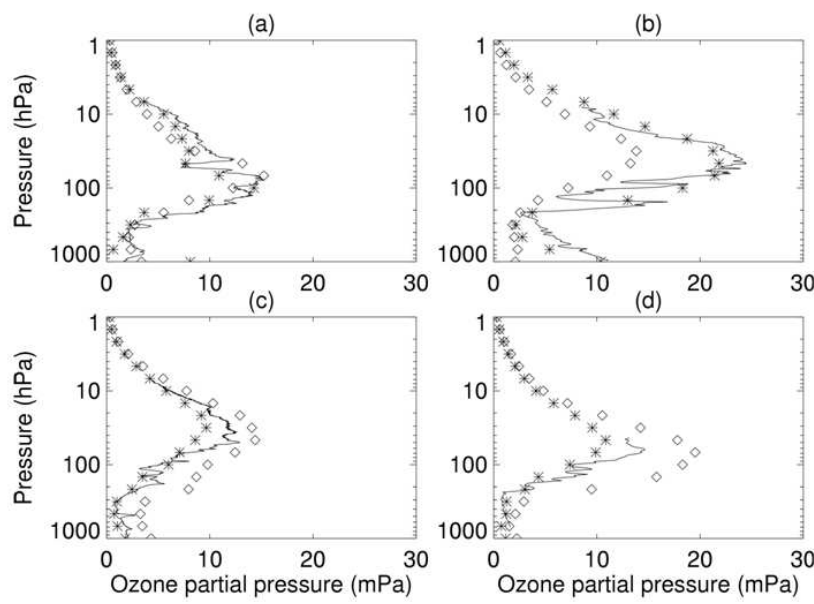

Fig. 3. Evaluation of ozone analyses using independent data at four locations: (a) Ny Alesund $\left(78.9^{\circ} \mathrm{N}, 11.9^{\circ} \mathrm{E}\right)$ on 27 April 1997; (b) Payerne $\left(46.8^{\circ} \mathrm{N}, 7.0^{\circ} \mathrm{E}\right.$ ) on 25 April 1997; (c) Lauder $\left(45.05^{\circ} \mathrm{S}\right.$, $\left.169.7^{\circ} \mathrm{E}\right)$ on 16 April 1997; and (d) South Pole $\left(90^{\circ} \mathrm{S}\right)$ on 18 April 1997; all plots at 12:00 UT. The analyses (stars) are compared against ozonesonde data (line) that have not been used in the assimilation. The ozone data used to initialize the assimilation are shown as diamonds. The results show reasonable agreement between the analyses and the ozonesondes, and the lack of influence of the initial ozone conditions after the spin-up period. Units are $\mathrm{mPa}$. With permission from Struthers et al. (2002).

averaging kernel information, which accounts for the information content, including the vertical resolution, of the observations (Migliorini et al., 2004). This is difficult in practice, as the averaging kernel information is not always readily supplied by the measuring instrument specifications.

In general, comparison against independent data is much more significant than comparison against the assimilated observations. Thus, independent data are the ultimate arbiter of the quality of analyses. In Sect. 6.1 we discuss the quality of humidity and ozone analyses from NWP- and CTM-based assimilation systems, based on the intercomparison of analyses between themselves and against independent data. We also mention briefly early efforts to carry out these intercomparisons for other stratospheric constituents

\subsection{Illustrative examples}

The use of constituent data assimilation to evaluate instruments is numerous. In the two examples below, data assimilation has been used to evaluate two scientific instruments onboard Envisat: GOMOS and MIPAS.

GOMOS is a stellar occultation instrument that measures, among other species, stratospheric night-time profiles of $\mathrm{O}_{3}$, $\mathrm{NO}_{2}$ and, for the first time, $\mathrm{NO}_{3}$. This last species has a very short life-time. During the day-time, its concentration is close to zero because it is photolysed in the presence of sunlight. During the night, its chemistry is very simple and

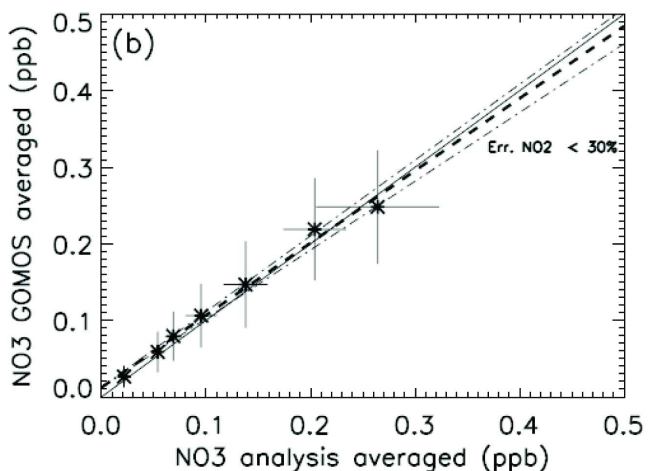

Fig. 4. GOMOS $\mathrm{NO}_{3}$ measurement and analysed $\mathrm{NO}_{3}$ averaged over isentropic levels; only data where the GOMOS $\mathrm{NO}_{2}$ error is below $30 \%$ are included. The isentropic levels included in the average are $735,900,990,1100,1210,1350$ and $1510 \mathrm{~K}$. The standard deviations of the isentropic means of GOMOS $\mathrm{NO}_{3}$ and of mean analysed $\mathrm{NO}_{3}$ are indicated by vertical and horizontal lines, respectively. With permission from Marchand et al. (2004).

strongly coupled to $\mathrm{O}_{3}$ and $\mathrm{NO}_{2}$. Marchand et al. (2004) have assimilated GOMOS $\mathrm{O}_{3}$ and $\mathrm{NO}_{2}$ in a photochemical box model using a variational approach. Showing good agreement between $\mathrm{NO}_{3}$ from GOMOS and the analyses, Marchand et al. (2004) validate the self-consistency of GOMOS $\mathrm{O}_{3}, \mathrm{NO}_{2}$ and $\mathrm{NO}_{3}$ measurements (see Fig. 4). It is also found that these GOMOS measurements are consistent with our current understanding of night-time $\mathrm{NO}_{3}$ chemistry.

Within the validation effort for MIPAS, Vigouroux et al. (2007) have compared MIPAS $\mathrm{N}_{2} \mathrm{O}$ and $\mathrm{HNO}_{3}$ with ground based FTIR measurements for 2003. They use a colocation criterion of $1000 \mathrm{~km}$ around ground-based stations within a time interval of $\pm 3 \mathrm{~h}$. In order to increase the number of co-locations, they also use MIPAS $\mathrm{N}_{2} \mathrm{O}$ and $\mathrm{HNO}_{3}$ analyses produced by the Belgian Assimilation System for Chemical Observations from Envisat, BASCOE. The standard deviation and bias between the co-located BASCOE analyses and FTIR observations are reduced from those between the co-located MIPAS and FTIR observations. This paper also discusses under what conditions these analyses can be considered a good proxy for MIPAS observations. In the case of $\mathrm{N}_{2} \mathrm{O}$, the agreement between BASCOE analyses and the MIPAS and FTIR data is excellent. Comparison with FTIR shows a bias ranging from $-5 \%$ to $+1 \%$, and standard deviations ranging from $2 \%$ to $7 \%$. Compared to the MIPAS random errors (Raspollini et al., 2006), these values are not significant. BASCOE appears to have more difficulty in producing proxies for MIPAS $\mathrm{HNO}_{3}$ profiles but the estimated standard deviations, less than $10 \%$ between BASCOE and FTIR, appear reasonable. 


\section{Applications}

In this section we provide examples of the application of data assimilation to evaluate analyses of stratospheric constituents; monitor the stratosphere; and provide ozone forecasts in near-real-time.

\subsection{Evaluation of analyses}

Objective evaluation of analyses can be obtained by the intercomparison of analyses produced using different data assimilation systems. If the systems assimilate a common observational dataset, differences between the analyses can be attributed to differences in the models and/or the data assimilation system. Furthermore, by confronting these analyses against others and against independent data (i.e., not assimilated) it is possible to both gain an understanding of their strengths and weaknesses, and to make new developments. Finally, these intercomparisons provide more information (and faster) than if each participant assessed their own system independently.

In this section we use the analyses intercomparison approach to assess the accuracies of humidity analyses in the stratosphere-mesosphere, Sect. 6.1.1 (Lahoz et al., 2007), and the accuracy of ozone analyses in the stratospheremesosphere, Sect. 6.1.2 (Geer et al. 2006a). Intercomparison of analyses of stratospheric constituents other than humidity and ozone are currently underway. For example, Errera et al. (2007) discusses the performance of $\mathrm{NO}_{2}$ analyses using the BASCOE chemical model and observations from MIPAS and GOMOS.

\subsubsection{Accuracy of humidity analyses}

The humidity analyses considered in some detail by Lahoz et al. (2007): BASCOE (CTM-based), and ECMWF and Met Office (NWP-based), have varying accuracies that depend on the assimilation system, the altitude and the latitude. Figure 5 shows the monthly mean zonal water vapour analyses for September 2003 for the ECMWF and BASCOE systems.

The monthly mean analyses show good agreement with the UARS reference atmosphere for September (http://code916.gsfc.nasa.gov/Public/Analysis/UARS/urap/ home.html). A number of well-known features can be seen in the stratospheric analyses from BASCOE and ECMWF. These include a relatively dry region above the tropical tropopause and dehydration of the Antarctic winter polar vortex (SPARC, 2000). The role of the Brewer-Dobson circulation on the distribution of water vapour is reflected in the upward and poleward propagation of the dry air entering the stratosphere through the tropical tropopause. Methane oxidation is responsible for the relatively moist upper stratosphere and lower mesosphere (see, e.g., LeTexier et al., 1988). The Brewer-Dobson circulation transports this moist air downwards within the winter hemisphere polar vortex.

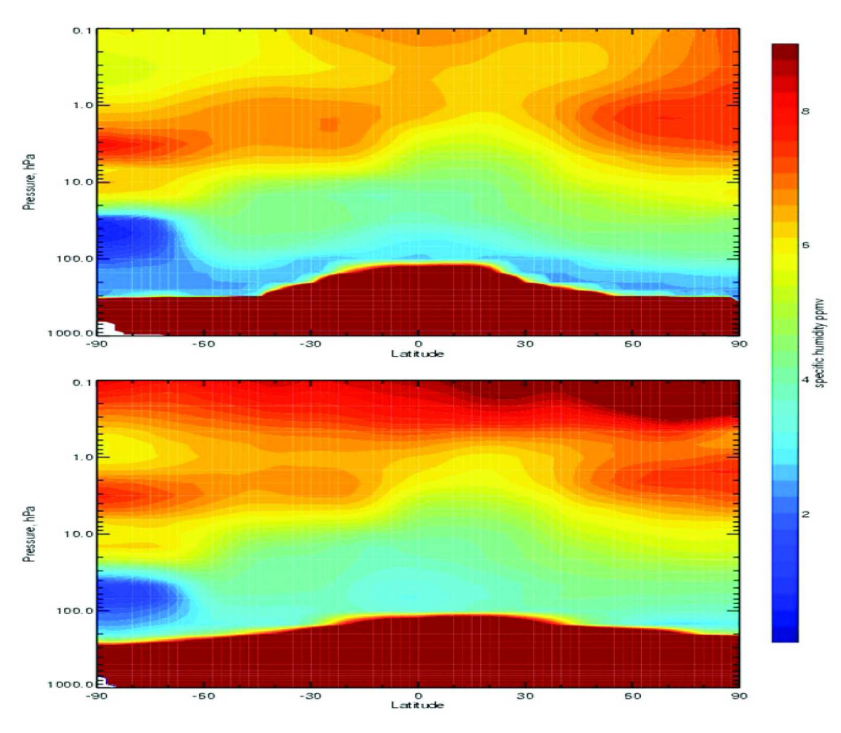

Fig. 5. Monthly zonal mean specific humidity analyses for September 2003 for BASCOE (upper plot) and ECMWF (lower plot). MIPAS water vapour profiles have been assimilated in both cases. Blue denotes relatively low specific humidity values; red denotes relatively high specific humidity values. Units: parts per million by volume, ppmv. Based on Lahoz et al. (2007).

Between the tropopause $(\sim 100 \mathrm{hPa})$ and $1 \mathrm{hPa}$, the zonal mean monthly analyses for the BASCOE and ECMWF systems are reasonably similar. The BASCOE analyses show a drier UTLS region at most latitudes, whereas the ECMWF analyses show a more distinct dry tropical tropopause region. Consequently, the vertical gradient in specific humidity in the lower stratosphere is stronger in the BASCOE analyses. The southern hemisphere polar vortex is drier in the BASCOE analyses. For levels above $1 \mathrm{hPa}$ the zonal mean specific humidity fields vary quite considerably between the two systems. In this region, the ECMWF analyses are $\sim 2$ ppmv (parts per million by volume) moister than the BASCOE analyses. The BASCOE analyses appear more realistic when compared to the UARS reference atmosphere. BASCOE analyses are $~ 5 \%$ lower than MIPAS data in the lower mesosphere, but the corresponding ECMWF analyses are $\sim 10 \%$ higher. However, the ECMWF analyses are $25 \%-30 \%$ too low compared to the uppermost MIPAS layer at $0.2 \mathrm{hPa}-0.1 \mathrm{hPa}$. It appears that the ECMWF analyses aim to find a compromise between these conflicting biases, as we might expect given that a vertical smoothing is imposed by the background error correlations. Most of the differences between the two analyses in the upper stratosphere/mesosphere and lower stratosphere can be explained by the fact that BASCOE does not assimilate any MIPAS data for levels below $95 \mathrm{hPa}$ and above $0.2 \mathrm{hPa}$ (data outside these regions are model generated). Influences from the troposphere and mesosphere are therefore excluded in the BASCOE model. 

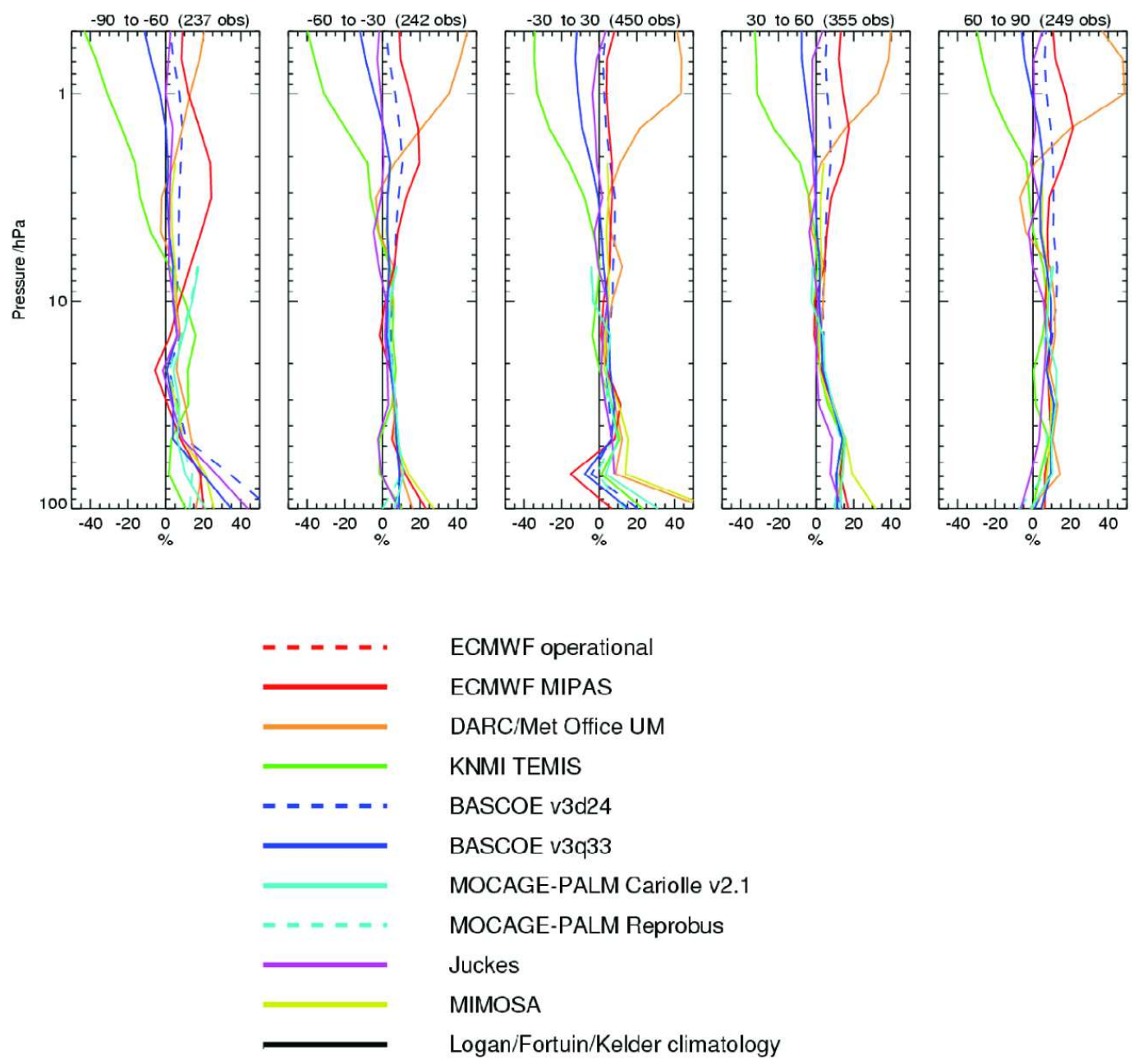

Fig. 6. Top: Mean of analysis minus HALOE differences (in percent), normalized by climatology, for the period 18 August-30 November 2003. Bottom: Colour key for top part of figure. The numbers in brackets indicate the HALOE/analysis coincidences within each latitude bin. Based on Geer et al. (2006a).

The Met Office has investigated the impact of varying the control variable in the assimilation of MIPAS humidity data. The objective is to develop a humidity control variable that has the desirable properties that it is usable in both the troposphere and the stratosphere; it has approximately Gaussian background errors; that temperature and humidity increments are decoupled; and that allows realistic vertical error correlations. To achieve this, the Met Office have combined the ideas of Dee and da Silva (2003) and Hólm et al. (2002), and defined a normalized relative humidity variable.

Lahoz et al. (2007) describe three different experiments by the Met Office where the humidity control variable is either relative humidity $(\mathrm{RH})$, normalized $\mathrm{RH}$ or normalized specific humidity. All three experiments show fairly reasonable specific humidity profiles for levels below $5 \mathrm{hPa}$. However, at higher levels the fit to the MIPAS observations is less good, with the analyses being consistently too dry. The experiment with the normalized specific humidity control variable has a more reasonable lower mesospheric specific humidity, but is still too dry when compared to the MIPAS observations. These results are still under study.

\subsubsection{Accuracy of ozone analyses}

The accuracy of ozone analyses from NWP- and CTMbased systems is discussed in detail in the intercomparison by Geer et al. (2006a). It is shown that the best performing analyses are capable of producing very good agreement with ozonesonde, HALOE and MIPAS ozone data. From the lower stratosphere to the lower mesosphere $(100 \mathrm{hPa}$ to $0.5 \mathrm{hPa}$ ), these analyses show biases less than $\pm 10 \%$ with respect to HALOE ozone data and ozonesondes. Standard deviations can be less than $10 \%$ above $50 \mathrm{hPa}$ and less than $20 \%$ in the lower stratosphere $(100 \mathrm{hPa}$ to $50 \mathrm{hPa})$. This shows that current assimilation techniques are capable of producing 
ozone analyses that have good agreement with independent data (see Fig. 6). These results are contingent upon the good quality of the assimilated ozone dataset: Dethof (2003) and Wargan et al. (2005) have already shown the benefits of MIPAS ozone over operational observations such as SBUV/2.

The enhanced skill of the best performing analyses can usually be attributed to better modelling of ozone chemistry or transport processes. The worse performing systems could often be easily improved by following similar modelling techniques. For example, this can apply to regions where there are limitations with the ozone data assimilated, where as shown by Geer et al. (2006a), CTMs and GCMs with chemistry generally do better. The intercomparison finds few differences that can be attributed to the assimilation technique or the model used (GCM or CTM). It would require focused experiments, rather than an intercomparison, to reveal such differences. Overall, the study by Geer et al. (2006a) shows that the first priority for ozone data assimilation systems is to improve the modelling of ozone chemistry and transport.

The work of Geer et al. (2006a, b) on the quality of ozone analyses has highlighted the importance of observational and model bias in DA. Besides providing information on observational bias, DA can provide information on, and be affected by, model bias. For example, Geer et al. (2006b), using the Met Office Unified Model, found that vertical transport of ozone in the tropical pipe, and transport in the BrewerDobson circulation, is much too fast as a result of known problems in the tracer transport scheme. This was manifested in that ozone forecasts above the ozone peak $(10 \mathrm{hPa})$ tended to be biased high against the MIPAS values (negative $\mathrm{OmF}$ values), and ozone forecasts around the ozone peak tended to be biased low against the MIPAS values (positive OmF values).

The Brewer-Dobson circulation is also degraded by problems with the assimilation of dynamical variables (Douglass et al., 2003; Schoeberl et al., 2003; Tan et al., 2004). This reflects that it is very hard for DA to handle slow processes, on timescales much longer than typical assimilation cycles. Problems with stratospheric tracer transport are seen in many DA systems (Oikonomou and O'Neill, 2006), and this remains a major focus of investigation.

Work by Monge-Sanz et al. (2007) shows that ECMWF ERA interim re-analyses (ECMWF 2007) can be used to provide realistic stratospheric transport over multi-annual timescales with an off-line CTM; in particular, the CTM's age of air agrees reasonably well with observations. The improvement, in comparison with forcing the CTM with ERA40 reanalyses or troposphere-stratosphere analyses from the Met Office, is attributed mainly to the use of 4D-Var (which uses observations at their correct time) and an improved balance operator, together leading to more balanced flow and reduced mixing in the subtropics. In addition, an improved implementation of the bias correction of satellite radiances is thought to have helped reduce the analysed strength of the Brewer-Dobson circulation.

Finally, several papers (Levelt et al., 1998; Chipperfield et al., 2002; Juckes, 2006, to name a few) show analysed constituent datasets that are closer to independent data than the assimilated observations or the simulated fields, thereby providing evidence that the DA method can add value to constituent information, either from observations or from a model. Jackson (2007) shows that assimilation of EOS MLS ozone data reduces mean analyses errors in the lower stratosphere. Compared to control simulations where no ozone data are assimilated, mean errors (evaluated against HALOE ozone data) dropped by $5 \%-25 \%$ in the Southern Hemisphere extra-tropics, and by $\sim 10 \%$ in the Northern Hemisphere extra-tropics; mean errors (evaluated against ozonesondes) dropped by $\sim 50 \%$ in the tropical UTLS.

Along these lines, Struthers et al. (2002) demonstrate that the combined assimilation of UARS MLS ozone profiles and GOME total column ozone gives analysed constituent datasets that are closer to independent data than either of the analyses derived from the assimilation of UARS MLS ozone profiles, or of GOME total column ozone. Thus, in this case, combined assimilation has added value to the single assimilation of these ozone datasets. Note, however, that this is not always the case, as there could be inconsistencies in the assimilation system, for instance in the treatment of biases (Rood, 2005). Thus, there is scope for improving the use of observations in constituent data assimilation.

\subsection{Stratospheric Ozone Monitoring}

Monitoring the stratosphere is done routinely by satellite instruments in order to track the evolution of the stratospheric composition, mainly ozone and the gases that destroy it (WMO, 2006). Currently, products from different data assimilation groups are used to help this monitoring effort and assess protocols.

ECMWF use their NWP operational system to monitor satellite ozone data by passive data assimilation, i.e., the ozone data are passed through the assimilation system and evaluated, but are not allowed to affect the analyses. For example, Dethof (2004) describes the monitoring of ozone profiles from the MIPAS and GOMOS instruments, and total column ozone from the SCIAMACHY instrument. As of October 2007, ECMWF assimilate operationally total ozone columns from SCIAMACHY and partial ozone columns from SBUV/2 on NOAA-16 (R. Dragani, personal communication, 2007). In an experimental suite, they also actively assimilate partial columns of ozone from SBUV/2 on NOAA17 and NOAA-18, and monitor passively OMI total column ozone, GOMOS ozone profiles, and total column ozone from the 9.7 micron channel of SEVIRI on MSG-9 (R. Dragani, personal communication, 2007). If the monitored data prove satisfactory, they are moved to active assimilation into the ECMWF operational system, and thus are allowed to affect 
the meteorological analyses (as well as the ozone analyses). For example, it is expected that once the evaluation of results from this experimental suite is satisfactory, ECMWF will start to assimilate operationally partial ozone columns from SBUV/2 on NOAA-17 and NOAA-18.

NCEP have set up an operational ozone monitoring and forecasting system within the NCEP Global Forecasting System (GFS). They use the CHEM2D-OPP chemistry module (McCormack et al., 2006). As of September 2007, the system assimilated several ozone products, including SBUV/2 partial ozone columns from NOAA-16 and NOAA-17, and total column ozone from OMI (Long et al., 2007).

Since 2000, KNMI produce near real time total ozone assimilation (Eskes et al., 2003). This system is constrained by total ozone observations provided by a variety of satellite instruments (GOME, SCIAMACHY or OMI, depending on the time period) and has delivered global maps of total ozone since August 1995 (http://www.temis.nl). This database is being used to evaluate the change of total ozone since the 1960s (WMO, 2006).

Stratospheric constituent assimilation using a full chemistry model and $4 \mathrm{D}-\mathrm{Var}$ is underway at DLR and BIRAIASB. In the framework of the ESA-funded PROMOTE project, these two institutions will provide re-analyses of stratospheric ozone from 1992 (i.e., soon after the launch of the UARS satellite) to the present, using ozone data from different sensors (see the Stratospheric Ozone Profile Record project, http://www.gse-promote.org for more details). In addition to ozone, they expect to provide analyses of several parameters related to ozone chemistry: $\mathrm{ClO}_{\mathrm{x}}, \mathrm{NO}_{\mathrm{x}}$, PSCs, ozone depletion rate and $\mathrm{Cl}_{\mathrm{y}}$ (total available chlorine). These re-analyses and analyses will be used by international organizations such as SPARC (Stratospheric Processes And their Role in Climate) in the framework of the Chemistry-Climate Model Validation (CCMVal) and WMOGAW (World Meteorological Organization - Global Atmospheric Watch) projects to assist in the evaluation of compliance with the Montreal protocol.

\subsection{Ozone forecasting}

Ozone forecasts are useful for predicting high UV-flux events. They can be used to warn populations near the Antarctic when the ozone hole moves above these areas or to warn populations near the Arctic when low ozone events (also known as ozone mini-holes) occur above these areas. They can also be used to plan observation campaigns. Ozone forecasts are operational at ECMWF since 2002 (Dethof, 2003), and operational at KNMI and GMAO since, respectively, 2000 (Eskes et al., 2003) and 1999 (Štajner et al., 2001).

The ECMWF products have been based on different ozone datasets, depending on their availability (see Sect. 6.2 for the status of operational ozone assimilation at ECMWF on October 2007). The KNMI products are based on to- tal column ozone measurements from the ESA instruments GOME and SCIAMACHY, and the NASA instrument OMI. GMAO products are based on TOMS total column ozone and SBUV/2 partial column ozone measurements. The ECMWF system is based on its NWP system, and includes parametrized ozone chemistry. The KNMI and GMAO systems are based on CTMs with parametrized ozone chemistry forced by off-line winds and temperature from, respectively, the ECMWF and GEOS models. Ozone forecasts are produced using the wind and temperature forecasts from the ECMWF and GEOS models.

BIRA-IASB also set up an ozone forecasting service using the BASCOE system (http://www.bascoe.oma.be). The system is based on a CTM with full chemistry and a scheme that explicitly calculates the microphysics of PSCs. The constraining observations are MIPAS near real time ozone profiles as well as five other chemical species $\left(\mathrm{NO}_{2}, \mathrm{HNO}_{3}\right.$, $\mathrm{N}_{2} \mathrm{O}, \mathrm{CH}_{4}$ and $\mathrm{H}_{2} \mathrm{O}$ ). In addition to ozone ten-day forecasts, this service also produced forecasts of $\mathrm{ClO}_{\mathrm{x}}, \mathrm{N}_{2} \mathrm{O}, \mathrm{HNO}_{3}$ and $\mathrm{ClONO}_{2}$ volume mixing ratio, and PSC surface area density. This service was operational for one and a half years, and ended in March 2004 when delivery of MIPAS near real time profiles was interrupted due to problems with the MIPAS instrument. This difficulty with the MIPAS instrument highlights the weakness of using near real time products from research instruments for operational services.

Eskes et al. (2002) estimate that useful ozone forecasts can be obtained up to about one week for the extra-tropics with the KNMI system. In the tropics, the forecast skill is less good (useful forecasts out to $\sim 2$ days) due, mainly, to the lack of tropospheric chemistry in the KNMI CTM. Two examples illustrate the skill of the KNMI system. The first concerns low ozone events that are observed during winter over the Atlantic and Northern Europe, and last for 1-2 days (Orsolini and Nikulin, 2006). These events are due to dynamical transport of low ozone from the subtropics to the extra-tropics. For these events, five-day ozone forecasts are found to be qualitatively good; three-day forecasts are found to be quantitatively equivalent to the analyses, the latter being close to the observations (GOME total column ozone). The second example concerns the Antarctic polar vortex split of September 2002. During this unprecedented event, associated with a stratospheric warming (Eskes et al., 2005), the vortex split into two parts before decaying. As a result of this, the ozone hole also split into two parts. Figure 7 shows the ozone total column on 26 September over Antarctica calculated by the KNMI analysis and 5-day, 7-day and 9-day forecasts of the total ozone column. The analysis for this day shows the ozone hole split with two distinct regions of low total column ozone (values less than 200 DU). For this event, forecasts out to seven days perform well, and differences from the analyses are small. The nine-day forecast captures elements of the ozone hole split.

These two cases highlight the maturity of the KNMI ozone forecast service. However, the high accuracy of the fore- 

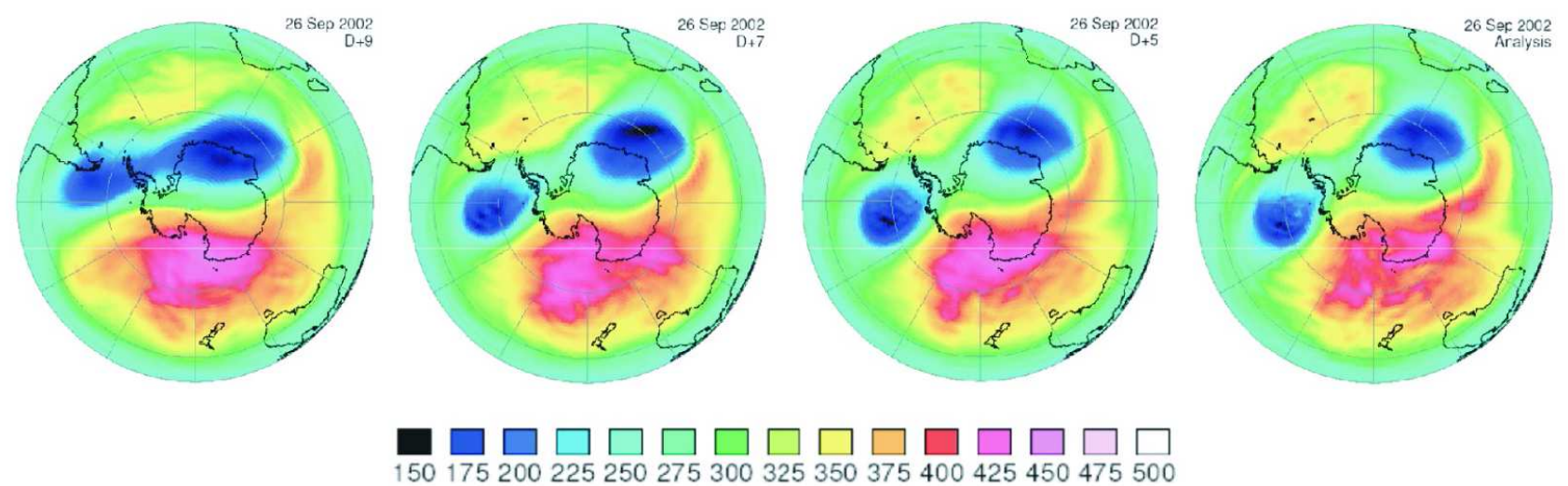

Fig. 7. Total column ozone on 26 September 2002, provided by the KNMI operational ozone assimilation system. From left to right: 9-day, 7-day, 5-day forecasts, and the corresponding analysis. With permission from Eskes et al. (2005).

casts would not have been possible without high quality dynamical fields, in this case from ECMWF. The success of the KNMI forecasts shows that the underlying dynamical processes were well captured by the ECMWF NWP system (Simmons et al., 2005).

\section{$7 \quad$ Future directions}

Stratospheric constituent data assimilation has developed enormously during the last 15 years to a position where incorporation of constituents in NWP (especially ozone) is routine. Two approaches have been used: GCM-based NWP models and chemical models, either CTMs or photochemical box models. Recently, the NWP and CTM approaches have started to be combined in coupled NWP/CTM data assimilation, e.g., in collaboration between Environment Canada, other Canadian partners and BIRA-IASB, where the Canadian GEM-strato GCM is coupled to the BASCOE CTM; early results are promising (Ménard et al., 2007). The CMAM data assimilation set-up at Met Service Canada (MSC) described by Polavarapu et al. (2005a, b) uses a GCM with full chemistry and can also be described as a coupled system.

These approaches to stratospheric constituent data assimilation have benefited from collaboration between operational and research institutions to identify shortcomings in the different assimilation approaches, for example within the EUfunded ASSET project (Lahoz et al., 2007) and the ASSET ozone intercomparison project (Geer et al., 2006a). The importance of maintaining and developing these collaborations has been noted (McLaughlin et al., 2005).

Key drivers in constituent data assimilation for the future are likely to include the need to monitor the environment (e.g. stratospheric ozone; tropospheric pollution); the need to comply with international treaties such as the Montreal protocol; and the need to comply with environmental legislation concerning, e.g., air quality. This is illustrated by the PROMOTE project (http://www.gse-promote.org), one of the GMES service elements set up by ESA. PROMOTE is a user-oriented project, which aims to use the assimilation of constituent data to provide services on global ozone, greenhouse gases and air quality.

Another area of increasing importance will be the relationship between chemistry and climate. While this is naturally mainly the focus of coupled chemistry-climate GCMs (see Eyring et al., 2006, and references therein), it does increase the importance of the compilation of assimilated constituent data for the study of recent climate variations and evaluation of climate simulations; climate/chemistry interactions will thus be one of the leading drivers for the development of coupled chemistry/dynamics assimilation systems. The inclusion of ozone in the recent ERA-40 re-analysis (Dethof and Hólm, 2004) illustrates the importance of these considerations. The EC and ESA initiative on GMES illustrates the perceived importance on more general environmental monitoring. The ECMWF-led GEMS project (Hollingsworth, 2005), part of GMES, illustrates the widening scope of data assimilation to include not just atmospheric dynamics but a widening range of atmospheric constituents.

In developing further constituent data assimilation for the stratosphere, choices will have to be made concerning issues such as the type of model, the complexity of the chemistry component in the model and the assimilation set-up. These choices will depend on the application (Lahoz, 2006). Challenges concerning issues such as bias, what datasets to assimilate, the need for ancillary datasets (e.g. aerosol information), representation of the model physics and chemistry, the suitability of the NWP approach, and the nature and evolution of the Global Observing System will have to be tackled. Insights gained in stratospheric constituent data assimilation will also help inform the challenges in tropospheric constituent data assimilation (Eskes, 2006). 
W. A. Lahoz et al.: Stratospheric constituent data assimilation

\section{Appendix A}

\section{Definition of acronyms}

$\begin{array}{ll}\text { ADEOS: } & \text { ADvanced Earth Observing Satellite } \\ \text { AIRS: } & \text { Atmospheric InfraRed Sounder } \\ \text { ASSET: } & \text { ASSimilation of Envisat daTa } \\ \text { ATMOS: } & \text { Atmospheric Trace MOlecule Spectroscopy } \\ \text { ATOVS: } & \text { Advanced TOVS } \\ \text { BASCOE: } & \begin{array}{l}\text { Belgian Assimilation System for Chemical Obser- } \\ \text { vations from Envisat }\end{array} \\ \text { BIRA- } & \text { Belgisch Instituut voor Rüimte } \\ \text { IASB: } & \end{array}$

$\begin{array}{ll}\text { BLUE: } & \text { Belgique } \\ \text { CCMVal: } & \text { Chemistry-Climate Model Validation } \\ \text { CLAES: } & \text { Cryogenic Limb Array Etalon Spectrometer } \\ \text { CMAM: } & \text { Canadian Middle Atmosphere Model } \\ \text { CRISTA: } & \begin{array}{l}\text { CRyogenic Infrared Spectrometers and Tele- } \\ \text { scopes for the Atmosphere }\end{array} \\ \text { CTM: } & \text { Chemistry-Transport Model } \\ \text { DA: } & \text { Data Assimilation } \\ \text { DARC: } & \text { Data Assimilation Research Centre, UK } \\ \text { DLR: } & \text { Deutsches zentrum für Luft-und Raumfahrt } \\ \text { DU: } & \text { Dobson Units } \\ \text { EC: } & \text { European Commission }\end{array}$

ECMWF: European Centre for Medium-range Weather Forecasts

EKF: $\quad$ Extended KF

EnKF: $\quad$ Ensemble KF

EOS: $\quad$ Earth Observing System

EOS MLS: EOS Microwave Limb Sounder

ERA: $\quad$ ECMWF Re-Analysis

ESA: $\quad$ European Space Agency

FGAT: $\quad$ First Guess at the Appropriate Time

FTIR: $\quad$ Fourier Transform InfraRed

GCM: General Circulation Model

GEOS: Goddard Earth Observing System

GEMS: Global Earth system Monitoring using Space and in-situ data

GEM- Global Environmental Multiscale (this model instrato: corporates the stratosphere)

GMAO: Global Modeling and Assimilation Office

GMES: Global Monitoring for Environment and Security

GOME and Global Ozone Monitoring Experiment

GOME-2:

GOMOS: Global Ozone Monitoring by Occultation of Stars

HALOE: HALogen Occultation Experiment

HIRS: $\quad$ High resolution Infrared Radiation Sounder

IASI: Infrared Atmospheric Sounding Interferometer

IGACO: Integrated Global Atmospheric Chemistry Observations

ILAS: $\quad$ Improved Limb Atmospheric Spectrometer

KF: Kalman Filter

KNMI: Koninklijk Nederlaands Meteorologisch Instituut

LIMS: $\quad$ Limb Infrared Monitor of the Stratosphere

MIPAS: Michelson Interferometer for Passive Atmo-

spheric Sounding

MLS: $\quad$ Microwave Limb Sounder

MSC: $\quad$ Met Service Canada

MSG: Meteosat Second Generation

NASA: National Aeronautics and Space Administration

NCAR: National Center for Atmospheric Research

NCEP: National Centers for Environmental Prediction

$\begin{array}{ll}\text { NCEP GFS: } & \text { NCEP Global Forecasting System } \\ \text { NMC: } & \text { National Meteorological Center } \\ \text { NOAA: } & \text { National Oceanic and Atmospheric Administra- } \\ & \text { tion } \\ \text { NWP: } & \text { Numerical Weather Prediction } \\ \text { OI: } & \text { Optimal Interpolation } \\ \text { OmA: } & \text { Observation minus Analysis } \\ \text { OmF: } & \text { Observation minus Forecast } \\ \text { OMI: } & \text { Ozone Monitoring Instrument } \\ \text { OSSE: } & \text { Observing System Simulation Experiment } \\ \text { POAM: } & \text { Polar Ozone and Aerosol Measurement } \\ \text { PROMOTE: } & \text { PROtocol MOniToring for the GMES service El- } \\ & \text { ement } \\ \text { PSAS: } & \text { Physical-space Statistical Analysis Scheme } \\ \text { PSC: } & \text { Polar Stratospheric Cloud } \\ \text { RH: } & \text { Relative Humidity } \\ \text { RT: } & \text { Radiative Transfer } \\ \text { SBUV/2: } & \text { Solar Backscatter Ultra-Violet/2 } \\ \text { SCIAMACHY: } & \text { Scanning Imaging Absorption spectrometer for } \\ & \text { Atmospheric CHartographY } \\ \text { SEVIRI: } & \text { Spinning Enhanced Visible and InfraRed Imager } \\ \text { SMR: } & \text { Sub-Millimeter Radiometer } \\ \text { SPARC: } & \text { Stratospheric Processes And their Role in Cli- } \\ & \text { mate } \\ \text { SSM/I: } & \text { Special Sensor Microwave/Imager } \\ \text { TIROS: } & \text { Television InfraRed Observation Satellite } \\ \text { TOMS: } & \text { Total Ozone Mapping Spectrometer } \\ \text { TOVS: } & \text { TIROS Operational Vertical Sounder } \\ \text { UARS: } & \text { Upper Atmosphere Research Satellite } \\ \text { UKMO: } & \text { UK Meteorological Office } \\ \text { UTLS: } & \text { Upper Troposphere/Lower Stratosphere } \\ \text { UV: } & \text { UltraViolet } \\ \text { Var: } & \text { Variational } \\ \text { WMO-GAW: } & \text { World Meteorological Organization - Global At- } \\ & \text { mospheric Watch } \\ & \\ & \end{array}$

Acknowledgements. During the preparation and submission of the ACPD version of this paper, WAL was funded by the NERC Data Assimilation Research Centre (DARC). He is now funded by the Norsk Institutt for Luftforskning (NILU), Norway. QE is supported by the Belgian Federal Science Policy in the framework of the BASCOE Prodex project; DF is funded by BIRA-IASB; RS by the Met Office, UK. Part of the work described in this review paper was funded by ASSET, an EU-funded Framework V project (contract EVK2-CT-2002-00137). We thank A. Geer, D. Jackson, B. Khattatov and O. Talagrand for commenting on earlier versions of this paper. We thank two anonymous referees and S. Eckermann for valuable comments that helped improve the paper. The International Space Science Institute (ISSI; http://www.issibern.ch) provided funds for a meeting to finish writing this paper.

Edited by: W. Ward

\section{References}

Anderson, J. L.: An ensemble adjustment filter for data assimilation, Mon. Weather Rev., 129, 2884-2903, 2001.

Auger, L. and Tangborn, A. V.: A wavelet-based reduced rank Kalman filter for assimilation of stratospheric chemical tracer observations, Mon. Weather Rev., 132, 1220-1237, 2004. 
Austin, J.: Towards the four-dimensional variational assimilation of stratospheric chemical constituents, J. Geophys. Res., 97, 25692588, 1992.

Austin, J., Barwell, B. R., Cox, S. J., et al.: The diagnosis and forecast of clear sky ultraviolet levels at the Earth's surface, Met. Apps., 1, 321-336, 1994.

Baier, F., Erbertseder, T., Morgenstern, O., et al.: Assimilation of MIPAS observations using a three-dimensional global chemistrytransport model, Q. J. Roy. Meteor. Soc., 131, 3529-3542, 2005.

Bertaux, J.-L., Kyrölä, E., and Wehr, T.: Stellar occultation technique for atmospheric ozone monitoring: GOMOS on Envisat, Earth. Observ. Q., 67, 17-20, 2000.

Blake, D. and Lindzen, R. S.: Effect of photochemical models on calculated equilibria and cooling rates in the stratosphere, Mon. Weather Rev., 101, 783-802, 1973.

Bormann, N. and Healy, S.: A fast radiative transfer model for the assimilation of infrared limb radiances from MIPAS: Accounting for horizontal gradients, Q. J. Roy. Meteor. Soc., 132, 23572376, 2006.

Bormann, N., Healy, S., and Hamrud, M.: Assimilation of MIPAS limb radiances in the ECMWF system. Part II: Experiments with a 2-dimensional observation operator, Q. J. Roy. Meteor. Soc., 133, 329-346, 2007.

Bormann, N., Matricardi, M., and Healy, S. B.: RTMIPAS: A fast radiative transfer model for the assimilation of infrared limb radiances from MIPAS, Q. J. Roy. Meteor. Soc., 131, 1631-1653, 2005.

Bormann, N. and Thépaut, J.-N.: Assimilation of MIPAS limb radiances in the ECMWF system. Part I: Experiments with a 1dimensional observation operator, Q. J. Roy. Meteor. Soc., 133, 309-327, 2007.

Bouttier, F. and Courtier, P.: Data assimilation concepts and methods. ECMWF training notes, March 1999, available from http: //www.ecmwf.int, 1999.

Bovensmann, H., Burrows, J. P., Buchwitz, M., et al.: SCIAMACHY: Mission objectives and measurement modes, J. Atmos. Sci., 56, 127-150, 1999.

Burrows, J. P., Weber, M., Buchwitz, M., et al.: The Global Ozone Monitoring Experiment (GOME): Mission Concept and First Scientific Results, J. Atmos. Sci., 56, 151-175, 1999.

Burrows, W. R., Vallée, M., Wardle, D. I., et al.: The Canadian operational procedure for forecasting total ozone and UV radiation, Met. Apps., 1, 247-265, 1994.

Caplan, P., Derber, J., Gemmill, W., et al.: Changes to the 1995 NCEP operational medium-range forecast model analysis - forecast system, Weather Forecast., 12, 581-594, 1997.

Cariolle, D. and Déqué, M.: Southern hemisphere medium-scale waves and total ozone disturbances in a spectral general circulation model, J. Geophys. Res., 91, 10 825-10 884, 1986.

Cariolle, D. and Morcrette, J.-J.: A linearized approach to the radiative budget of the stratosphere: influence of the ozone distribution, Geophys. Res. Lett., 33, L05806, doi:10.1029/2005GL025597, 2006.

Cariolle, D. and Teyssèdre, H.: A revised linear ozone photochemistry parameterization for use in transport and general circulation models: Multi-annual simulations, Atmos. Chem. Phys., 7, 2183-2196, 2007,

http://www.atmos-chem-phys.net/7/2183/2007/.

Carlotti, M., Dinelli, B. M., Raspollini, P., and Ridolfi, M.: Geo- fit approach to the analysis of limb-scanning satellite measurements, Appl. Opt., 41, 1872-1885, 2001.

Chapnik, B., Desroziers, G., Rabier, F., and Talagrand, O.: Diagnosis and tuning of observational error statistics in a quasioperational data assimilation setting, Q. J. Roy. Meteor. Soc., 132, 543-565, 2006.

Chipperfield, M. P., Khattatov, B. V., and Lary, D. J.: Sequential estimation of stratospheric chemical observations in a three-dimensional model, J. Geophys. Res., 107, 4585, doi:10.1029/2002JD002110, 2002.

Cohn, S. E.: Dynamics of short-term univariate forecast error covariances, Mon. Weather Rev., 121, 3123-3149, 1993.

Cohn, S. E.: An introduction to estimation theory, J. Meteorol. Soc. Jpn., 75, 257-288, 1997.

Cohn, S. E., da Silva, A., Guo, J., et al.: Assessing the effects of data selection with the DAO physical-space statistical analysis system, Mon. Weather Rev., 126, 2913-2926, 1998.

Collins, W. J., Rasch, P. J., Eaton, B. E., et al.: Simulating aerosols using a chemical transport model with assimilation of satellite retrievals: Methodology for INDOEX, J. Geophys. Res., 106, 7313-7336, 2001.

Connew, P.: Chemical data assimilation using the UKMO Unified Model, Procedings of the SODA workshop on Chemical Data Assimilation, 9-10 December 1998, KNMI, De Bilt, Netherlands, 1999.

Courtier, P.: Dual formulation of four-dimensional variational assimilation, Q. J. Roy. Meteor. Soc., 123, 2449-2461, 1997.

Coy, L., Allen, D. R., Eckermann, S. D., et al.: Effects of model chemistry and data biases on stratospheric ozone assimilation, Atmos. Chem. Phys., 7, 2917-2935, 2007, http://www.atmos-chem-phys.net/7/2917/2007/.

Daley, R.: Estimating the wind field from chemical constituent observations: Experiments with a one-dimensional Extended Kalman Filter, Mon. Weather Rev., 123, 181-198, 1995.

Davies, T., Cullen, M. J. P., Malcolm, A. J., et al.: A new dynamical core for the Met Office's global and regional modelling of the atmosphere, Q. J. Roy. Meteor. Soc., 131, 1759-1782.

Dee, D. P.: Bias and data assimilation, Q. J. Roy. Meteor. Soc., 131, 3323-3343, 2005.

Dee, D. and da Silva, A.: Data assimilation in the presence of forecast bias, Q. J. Roy. Meteor. Soc., 124, 269-295, 1998.

Dee D. and de Silva, A.: The choice of variable for atmospheric moisture analysis, Mon. Weather Rev., 131, 155-171, 2003.

Derber, J., Pan, H.-L., Alpert, J., et al.: Changes to the 1998 NCEP Operational MRF Model Analysis/Forecast system, available from http://www.nws.noaa.gov/om/tpb/449/449body.htm, 1998.

Derber, J. C. and Wu, W.-S.: The use of TOVS cloud-cleared radiances in the NCEP SSI analysis system, Mon. Weather Rev., 126, 2287-2299, 1998.

Desroziers, G., Berre, L., Chapnik, B., and Poli, P.: A simple method to diagnose and adapt observation and background errors, Q. J. Roy. Meteor. Soc., 131, 3385-3396, 2005.

Dessler, A. E.: The Chemistry and Physics of Stratospheric Ozone, Academic Press, 224 pp., 2000.

Dethof, A.: Assimilation of ozone retrievals from the MIPAS instrument onboard ENVISAT, ECMWF Tech Memo 428, 2003.

Dethof, A.: Monitoring and assimilation of MIPAS, SCIAMACHY and GOMOS retrievals at ECMWF. ESA Contract 17585/03/IOL: Technical support for global validation of ENVISAT data 
products, 2004.

Dethof, A. and Hólm, E.: Ozone assimilation in the ERA-40 reanalysis project, Q. J. Roy. Meteor. Soc., 130, 2851-2872, 2004.

Douglass, A. R., Schoeberl, M. R., Rood, R. B., and Pawson, S.: Evaluation of transport in the lower tropical stratosphere in a global chemistry and transport model, J. Geophys. Res., 108, 4529, doi:10.1029/2002JD002696, 2003.

ECMWF: ECMWF Newsletter No. 100 - Winter 2006/07, available from http://www.ecmwf.int, 2007.

El Amraoui, L., Ricaud, P., Urban, J., et al.: Assimilation of ODIN/SMR $\mathrm{O}_{3}$ and $\mathrm{N}_{2} \mathrm{O}$ measurements in a three-dimensional chemistry transport model, J. Geophys. Res., 109, D22304, doi:10.1029/2004JD004796, 2004.

Elbern, H., Strunk, A., Schmidt, H., and Talagrand, O.: Emission rate and chemical state estimation by 4-dimensional variational inversion, Atmos. Chem. Phys., 7, 3749-3769, 2007, http://www.atmos-chem-phys.net/7/3749/2007/.

El Serafy, G. Y. and Kelder, H. M.: Near-real-time approach to assimilation of satellite-retrieved 3D ozone fields in a global model using a simplified Kalman filter, Q. J. Roy. Meteor. Soc., 129, 3099-3120, 2003.

El Serafy, G. Y., van der A, R. J., Eskes, H., and Kelder, H. M.: Assimilation of 3D ozone field in global chemistry transport models using the full Kalman filter, Adv. Space Res., 30, 2473-2478, 2002.

Errera, Q., Bonjean, S., Chabrillat, S., et al.: BASCOE assimilation of ozone and nitrogen dioxide observed by MIPAS and GOMOS: Comparison between the two sets of analyses, ESA Special Publication SP-636, 2007.

Errera, Q. and Fonteyn, D.: Four-dimensional variational chemical data assimilation of CRISTA stratospheric measurements, J. Geophys. Res., 106, 12 253-12 265, 2001.

Eskes, H. J.: The integration of atmospheric chemistry observations by next generation global/hemispheric and regional and NWP models, in: Chemical Data Assimilation for the Observation of the Earth's Atmosphere, ACCENT/WMO Expert Workshop in support of IGACO, edited by: Barrie, L. A., Burrows, J. P., Monks, P., and Borrell, P., WMO Tech, Report 1360, GAW Report 169, 44-49, 2006.

Eskes, H. J., Segers, A., and van Velthoven, P. F. J.: Ozone forecasts of the Stratospheric Polar Vortex-Splitting Event in September 2002, J. Atmos. Sci., 62, 812-821, 2005.

Eskes, H. J., van Velthoven, P. F. J., and Kelder, H. M.: Global ozone forecasting based on ERS-2 GOME observations, Atmos. Chem. Phys., 2, 271-278, 2002, http://www.atmos-chem-phys.net/2/271/2002/.

Eskes, H. J, van Velthoven, P. F. J., Valks, P. J. M., and Kelder, H. M.: Assimilation of GOME total-ozone satellite observations in a three-dimensional tracer-transport model, Q. J. Roy. Meteor. Soc., 129, 1663-1681, 2003.

Evensen, G.: The Ensemble Kalman filter: theoretical formulation and practical implementation, Ocean Dyn., 53, 343-367, 2003.

Eyring, V., Butchart, N., Waugh, D. W., et al.: Assessment of temperature, trace species, and ozone in chemistry-climate model simulations of the recent past, J. Geophys. Res., 111, D22308, doi:10.1029/2006JD007327, 2006.

Fierli, F., Hauchecorne, A., Bekki, S., et al.: Data assimilation of stratospheric ozone using a high-resolution transport model, Geophys. Res. Lett., 29, 7381, doi:10.1029/2001GL014272,
2002.

Fischer, H., Blom, C., Oelhaf, H., et al.: Envisat MIPAS - An instrument for atmospheric chemistry and climate research, edited by: Readings, C. and Harris, R. A., ESA Publication SP-1229, 2000.

Fisher, M.: Background error covariance modelling. In ECMWF Seminar Recent developments in data assimilation for atmosphere and ocean, September 2003, available from http://www. ecmwf.int, 2003.

Fisher, M. and Andersson, E.: Developments in 4D-Var and Kalman filtering, ECMWF Tech Memo 347, 2001.

Fisher, M. and Lary, D. J.: Lagrangian 4-dimensional variational data assimilation of chemical species, Q. J. Roy. Meteor. Soc., 121, 1681-1704, 1995.

Fonteyn D., Errera, Q., DeMazière, M., et al.: 4D-Var assimilation of stratospheric aerosol satellite data, Adv. Space Res., 26, 2049 2052, 2001.

Fortuin, J. P. F and Kelder, H. L.: An ozone climatology based on ozonesonde and satellite measurements, J. Geophys. Res., 103, 31 709-31 734, 1998.

Geer, A. J., Lahoz, W. A., Bekki, S., et al.: The ASSET intercomparison of ozone analyses: method and first results, Atmos. Chem. Phys., 6, 5445-5474, 2006a.

Geer, A. J., Lahoz, W. A., Jackson, D. R., et al.: Evaluation of linear ozone photochemistry parametrizations in a stratospheretroposphere data assimilation system, Atmos. Chem. Phys., 7, 939-959, 2007, http://www.atmos-chem-phys.net/7/939/2007/.

Geer, A. J., Peubey, C., Bannister, R., et al.: Assimilation of stratospheric ozone from MIPAS into a global general circulation model: the September 2002 vortex split, Q. J. Roy. Meteor. Soc., 132, 231-257, 2006b.

Gille, J. C. and Russell, J. M.: The Limb Infrared Monitor of the Stratosphere: Experiment Description, Performance, and Results, J. Geophys. Res., 89, 5125-5140, 1984.

Gunson, M. R., Abbas, M. M., Abrams, M. C., et al.: The Atmospheric Trace Molecule Spectroscopy (ATMOS) experiment: Deployment on the ATLAS Space Shuttle missions, Geophys. Res. Lett., 23, 2333-2336, 1996.

Hadjinicolau, P., Pyle, J. A., Chipperfield, M. P., and Kettleborough, J. A.: Effect of interannual meteorological variability on mid latitude $\mathrm{O}_{3}$, Geophys. Res. Lett., 24, 2993-2996, 1997.

Hollingsworth, A.: Global Earth-system Modelling using Space and in situ data. ECMWF seminar proceedings, September 2005, available from http://www.ecmwf.int, 2005.

Hólm, E., Andersson, E., Beljaars, A., et al.: Assimilation and modelling of the hydrological cycle: ECMWF's status and plans, ECMWF Tech Memo 383, 2002.

Ide, K., Courtier, P., Ghil, M., and Lorenc, A.: Unified notation for data assimilation: Operational sequential and variational, J. Meteorol. Soc. Jpn., 75, 181-189, 1997.

IGACO: The Changing Atmosphere. An Integrated Global Atmospheric Chemistry Observation theme for the IGOS partnership, ESA SP-1282, Report GAW No. 159 (WMO TD No. 1235), September 2004, Implementation up-date, December 2004, available from: http://www.igospartners.org/docsTHEM. htm, 2004.

Jackson, D. R.: Improvements in data assimilation at the Met Office. Forecasting Research Technical Report No. 454, Met Office, 
2004.

Jackson, D. R.: Assimilation of EOS MLS ozone observations in the Met Office Data Assimilation System, Q. J. Roy. Meteor. Soc., 133, 1771-1788, 2007.

Jackson, D. R. and Saunders, R.: Ozone data assimilation: Preliminary system, Forecasting Research Technical Report No. 394, Met Office, 2002.

Joiner, J., Lee, H.-T., Strow, L. L., et al.: Radiative transfer in the 9.6 $\mu \mathrm{m}$ HIRS ozone channel using collocated SBUV-determined ozone abundances, J. Geophys. Res., 103, 19213-19230, 1998.

Juckes, M. N.: Evaluation of MIPAS ozone fields assimilated using a new algorithm constrained by isentropic tracer advection, Atmos. Chem. Phys., 6, 1549-1565, 2006, http://www.atmos-chem-phys.net/6/1549/2006/.

Kalnay, E.: Atmospheric Modeling, Data Assimilation and Predictability, Cambridge University Press, 341 pp., 2003.

Khattatov, B. V.: Multivariate chemical data assimilation, in: Data Assimilation for the Earth System, NATO ASI Series, edited by: Swinbank, R., Shutyaev, V. and Lahoz, W. A., Kluwer, 279-288, 2003.

Khattatov, B. V., Gille, J. C., Lyjak, L. V., et al.: Assimilation of photochemically active species and a case analysis of UARS data, J. Geophys. Res., 104, 18 715-18 737, 1999.

Khattatov, B. V., Lamarque, J.-F., Lyjak, L. V., et al.: Assimilation of satellite observations of long-lived chemical species in global chemistry transport models, J. Geophys. Res., 105, 29 135-29 144, 2000.

Khattatov, B., Lyjak, L., and Gille, J.: On applications of photochemical models to the design of measurement strategies, Geophys. Res. Lett., 28, 2377-2380, 2001.

Kistler, R., Kalnay, E., Collins, W., et al.: The NCEP-NCAR 50year reanalysis: Monthly means CD-ROM and documentation, B. Am. Meteorol. Soc., 82, 247-267, 2001.

Küll, V., Riese, M., Tie, X., et al.: $\mathrm{NO}_{\mathrm{y}}$ partitioning and aerosol influences in the stratosphere, J. Geophys. Res., 107, 8183, doi:10.1029/2001JD00001246, 2002.

Lahoz, W. A.: Chemical data assimilation: Choices and challenges, in: Chemical Data Assimilation for the Observation of the Earth's Atmosphere, ACCENT/WMO Expert Workshop in support of IGACO, edited by: Barrie, L. A., Burrows, J. P., Monks, P., and Borrell, P., WMO Tech, Report 1360, GAW Report 169, 106-110, 2006.

Lahoz, W. A., Brugge, R., Jackson, D. R., et al.: An Observing System Simulation Experiment to evaluate the scientific merit of wind and ozone measurements from the future SWIFT instrument, Q. J. Roy. Meteor. Soc., 131, 503-523, 2005.

Lahoz, W. A., Geer, A. J., Bekki, S., et al.: The Assimilation of Envisat data (ASSET) project, Atmos. Chem. Phys., 7, 17731796, 2007, http://www.atmos-chem-phys.net/7/1773/2007/.

Lary, D. J.: Data assimilation: a powerful tool for atmospheric chemistry, Phil. Trans. R. Soc. Lond., A357, 3445-3457, 1999.

Lary, D. J., Khattatov, B., and Mussa, H. Y.: Chemical data assimilation: A case study of solar occultation data from the ATLAS 1 mission of the Atmospheric Trace Molecule Spectroscopy Experiment (ATMOS), J. Geophys. Res., 108, 4556, doi:10.101029/2003JD003500, 2003.

Lemus-Deschamps, L., Grainger, S., Rikus, L., et al.: Australian $\mathrm{UV}$ and ozone forecasting system, available from http://www. bom.gov.au/bmrc/mdev/expt/uvindex/uvi.shtml, 2005.

LeTexier, H., Solomon, S., and Garcia, R. R.: The role of molecular hydrogen and methane oxidation in the water vapour of the stratosphere, Q. J. Roy. Meteor. Soc., 114, 281-295, 1988.

Levelt, P. F., Khattatov, B. V., Gille, J. C., et al.: Assimilation of MLS ozone measurements in the global three-dimensional chemistry transport model ROSE, Geophys. Res. Lett., 25, 4493 4496, 1998.

Levelt, P. F., van den Oord, G. H. J., Dobber, M. R., et al.: The Ozone Monitoring Instrument, IEEE Trans. Geosci. Remote Sensing, 44, 1093-1101, 2006.

Lindzen, R. S. and Goody, R.: Radiative and photochemical processes in mesospheric dynamics: Part I, models for radiative and photochemical processes, J. Atmos. Sci., 22, 341-348, 1965.

Logan, J. A.: An Analysis of Ozonesonde Data for the Troposphere: Recommendations for Testing 3-D Models, and Development of a Gridded Climatology for Tropospheric Ozone, J. Geophys. Res., 104, 16 115-16 149, 1999.

Long, C. S.: UV Index forecasting practices around the world, SPARC Newsletter No. 21, June 2003.

Long, C. S., Zhu, S., and Treadon, R.: Assimilation of multiple ozone products into the NCEP operational forecast model, Presentation at the SPARC Data Assimilation Workshop, Toronto, September 2007, abstract available from http://atlas-conferences. com/c/a/u/e/10.htm, 2007.

Lorenc, A. C., Roulstone, I., and White, A.: On the choice of control fields in var, Forecasting Research Technical Report No. 419, The Met Office, 2004.

Louvel, S.: Implementation of a dual variational algorithm for assimilation of synthetic altimeter data in the oceanic primitive equation model MICOM, J. Geophys. Res., 106, 9199-9212, 2001.

Lucke, R. L., Kowan, D., Bevilacqua, R. M., et al.: The Polar Ozone and Aerosol Measurement (POAM III) instrument and early validation results, J. Geophys. Res., 104, 18 785-18 799, 1999.

Lyster, P. M., Cohn, S. E., Ménard, R., et al.: Parallel implementation of a Kalman filter for constituent data assimilation, Mon. Weather Rev., 125, 1674-1686, 1997.

McCormack, J. P., Eckermann, S. D., Coy, L., et al.: NOGAPSALPHA model simulations of stratospheric ozone during the SOLVE2 campaign, Atmos. Chem. Phys., 4, 2401-2423, 2004, http://www.atmos-chem-phys.net/4/2401/2004/.

McCormack, J. P., Eckermann, S. D., Siskind, D. E., and McGee, T. J.: CHEM2D-OPP: A new linearized gas-phase ozone photochemistry parameterization for high-altitude NWP and climate models, Atmos. Chem. Phys., 6, 4943-4972, 2006, http://www.atmos-chem-phys.net/6/4943/2006/.

McLaughlin, D., O’Neill, A., Derber, J., and Kamachi, M.: Opportunities for enhanced collaboration within the data assimilation community, Q. J. Roy. Meteor. Soc., 131, 3683-3693, 2005.

McLinden, C. A., Olsen, S. C., Hannegan, B., et al.: Stratospheric ozone in 3-D models: A simple chemistry and the crosstropopause flux, J. Geophys. Res., 105, 14 653-14 665, 2000.

McNally, A. P., Watts, P., Smith, J., et al.: The assimilation of AIRS radiance data at ECMWF, Q. J. Roy. Meteor. Soc., 132, 935-958, 2006.

McPeters, R. D., Bhartia, P. K., Krueger, A., et al.: Earth Probe Total Ozone Mapping Spectrometer (TOMS) Data Products User's Guide, NASA Reference Publication 1998-206895, 1998. 
Marchand, M., Bekki, S., Denis, L., and Pommereau, J.-P.: Test of the nightime polar stratospheric $\mathrm{NO}_{2}$ decay using wintertime SAOZ measurements and chemical data assimilation, Geophys. Res. Lett., 30, 1920, doi:10.1029/2003GL017582, 2003.

Marchand, M., Bekki, S., Hauchecorne, A., and Bertaux, J.-L.: Validation of the self-consistency of GOMOS $\mathrm{NO}_{3}, \mathrm{NO}_{2}$ and $\mathrm{O}_{3}$ data using chemical data assimilation, Geophys. Res. Lett., 31, L10107, doi:10.1029/2004GL019631, 2004.

Massart, S., Cariolle, D., and Peuch, V.-H.: Towards an improvement of the atmospheric ozone distribution and variability by the assimilation of satellite data, C. R. Geosciences, 15, 1305-1310, 2004.

Mathison, C., Jackson, D. R., and Keil, M.: Methods of improving the representation of ozone in the Met Office model, NWP Tech. Report No. 502, Met Office, 2007.

Ménard, R., Chabrillat, S., Charette, C., et al.: Coupled chemistrydynamics data assimilation, Presentation at the SPARC Data Assimilation Workshop, Toronto, September 2007, abstract available from http://atlas-conferences.com/c/a/u/e/25.htm, 2007.

Ménard, R. and Chang, L.-P.: Stratospheric assimilation of chemical tracer observations using a Kalman filter, part II: Chi-squared validated results and analysis of variance and correlation dynamics, Mon. Weather Rev., 128, 2672-2686, 2000.

Ménard, R., Cohn, S. E., Chang, L. P., and Lyster, P. M.: Stratospheric assimilation of chemical tracer observations using a Kalman filter, part I: Formulation, Mon. Weather Rev., 128, 2654-2671, 2000.

Migliorini, S., Piccolo, C., and Rodgers, C. D.: Intercomparison of direct and indirect measurements: MIPAS versus sonde ozone profiles, J. Geophys. Res., 109, D19316, doi:10.1029/2004JD004988, 2004.

Miller, A. J., Nagatani, R. M., Flynn, L. E., et al.: A cohesive total ozone data set from the SBUV(/2) satellite system, J. Geophys. Res., 107, 4701, doi:10.1029/2001JD000853, 2002.

Molod, A., Helfand, H. M., and Takacs, L. L.: The climatology of parameterized physical processes in the GEOS-1 GCM and their impact on the GEOS-1 data assimilation system, J. Climate, 9, 764-785, 1996.

Monge-Sanz, B. M., Chipperfield, M. P., Simmons, A. J., and Uppala, S. M.: Mean age of air and transport in a CTM: Comparison of different ECMWF analyses, Geophys. Res. Lett., 34, L04801, doi:10.1029/2006GL028515, 2007.

Morcrette, J.-J.: Ozone radiation interactions in the ECMWF forecast system, ECMWF Tech Memo 375, 2003.

Müller, M. D., Bhartia, P. K., and Štajner, I.: Assimilation of SBUV version 8 radiances into the GEOS DAS. Proceedings of the Quadrennial Ozone Symposium, GOS, Kos, Greece, June 2004, available from ftp://gmaoftp.gsfc.nasa.gov/ pub/papers/ivanka/ozone_papers/QOS04.Mueller.pdf, 2004.

Murtagh, D., Fisk, U., Merino, F., et al.: An overview of the Odin atmospheric mission, Can. J. Phys., 80, 309-319, 2002.

Nakajima, H., Sugita, T., Yokota, T., et al.: Characteristics and performance of the Improved Limb Atmospheric SpectrometerII (ILAS-II) on board the ADEOS-II satellite, J. Geophys. Res., 111, D11501, doi:10.1029/2005JD006334, 2006.

Offermann, D., Grossman, K.-U., Barthol, P., et al.: The cryogenic infrared spectrometer and telescopes for the atmosphere (CRISTA) experiment and middle atmosphere variability, J. Geophys. Res., 104, 16311-16327, 1999.
Oikonomou, E. and O'Neill, A.: An evaluation of water vapour and ozone in the ERA-40 reanalysis compared with UARS data, J. Geophys. Res., 111, D14109, doi:10.1029/2004JD005341, 2006.

Orsolini, Y. J. and Nikulin, G.: A low-ozone episode during the European heat wave of August 2003, Q. J. Roy. Meteor. Soc., 132, 667-680, 2006.

Ott, E., Hunt, B. R., Szunyogh, I., et al.: A local ensemble Kalman filter for atmospheric data assimilation, Tellus, 56A, 415-428, 2004.

Parrish, D. and Derber, J. C.: The National Meteorological Center's spectral statistical interpolation analysis system, Mon. Weather Rev., 120, 1747-1763, 1992.

Peuch, A., Thépaut, J.-N., and Pailleux, J.: Dynamical impact of total-ozone observations in a four-dimensional variational assimilation, Q. J. Roy. Meteor. Soc., 126, 1641-1659, 2000.

Pham, D. T.: Stochastic methods for sequential data assimilation in strongly nonlinear systems, Mon. Weather Rev., 129, 11941207, 2001.

Plumb, R. A. and Ko, M. K. W.: Interrelationships between mixing ratios of long-lived stratospheric constituents, J. Geophys. Res., 97, 10 145-10 156, 1992.

Polavarapu, S., Ren, S., Rochon, Y., et al.: Data assimilation with the Canadian Middle Atmosphere Model, Atmos.-Ocean, 43, 77-100, 2005a.

Polavarapu, S., Shepherd, T. G., Rochon, Y., and Ren, S.: Some challenges of middle atmosphere data assimilation, Q. J. Roy. Meteor. Soc., 131, 3513-3527, 2005 b.

Raspollini, P., Belotti, C., Burgess, A., et al.: MIPAS level 2 operational analyses, Atmos. Chem. Phys., 6, 5605-5630, 2006, http://www.atmos-chem-phys.net/6/5605/2006/.

Rawlins, F., Ballard, S. P., Bovis, K. J., et al.: The Met Office global four-dimensional variational data assimilation scheme, Q. J. Roy. Meteor. Soc., 133, 347-362, 2007.

Riishøjgaard, L.-P.: On four-dimensional variational assimilation of ozone data in weather-prediction models, Q. J. Roy. Meteor. Soc., 122, 1545-1572, 1996.

Riishøjgaard, L.-P.: A direct way of specifying flow-dependent background error correlations for meteorological analysis systems, Tellus, 50, 42-57, 1998.

Riishøjgaard, L.-P., Štajner, I., and Lou, G.-P.: The GEOS ozone data assimilation system, Adv. Space Res., 25, 1063-1072, 2000.

Roche, A. E., Kumer, J. B., Mergenthaler, J. L., et al.: The Cryogenic Limb Array Etalon Spectrometer (CLAES) on UARS: Experiment Description and Performance, J. Geophys. Res., 98, 10763-10 775, 1993.

Rodgers, C. D.: Inverse Methods for Atmospheric Sounding: Theory and Practice, World Scientific Publishing Co., Singapore, 238 pp., 2000.

Rood, R. B.: Ozone assimilation, in: Data Assimilation for the Earth System, NATO ASI Series, edited by: Swinbank, R., Shutyaev, V. and Lahoz, W. A., Kluwer, 263-277, 2003.

Rood, R. B.: Assimilation of stratospheric meteorological and constituent observations: A Review, SPARC Newsletter No. 25, July 2005.

Rösevall, J. D., Murtagh, D. P., and Urban, J.: Ozone depletion in the 2006/2007 Arctic winter, Geophys. Res. Lett., 34, L21809, doi:10.1029/2007GL030620, 2007a.

Rösevall, J. D., Murtagh, D. P., Urban, J., and Jones, A. K.: A study of polar ozone depletion based on sequential assimilation 
of satellite data from the ENVISAT/MIPAS and Odin/SMR instruments, Atmos. Chem. Phys., 7, 899-911, 2007b.

Russell III, J. M., Gordley, L. L., Park, J. H., et al.: The Halogen Occultation Experiment, J. Geophys. Res., 98, 10777-19797, 1993.

Sassi, F., Boville, B. A., Kinnison, D., and Garcia, R. R.: The effects of interactive ozone chemistry on simulations of the middle atmosphere, Geophys. Res. Lett., 32, L07811, doi:10.1029/2004GL022131, 2005.

Saunders, R., Matricardi, M., and Brunel, P.: An improved fast radiative transfer model for assimilation of satellite radiance observations, Q. J. Roy. Meteor. Soc., 125, 1407-1426, 1999.

Schoeberl, M. R., Douglass, A. R., Zhu, Z., and Pawson, S.: A comparison of the lower stratospheric age spectra derived from a general circulation model and two data assimilation systems, J. Geophys. Res., 108, 4113, doi:10.1029/2002JD002652, 2003.

Segers, A. J., Eskes, H. J., van der A, R. J., et al.: Assimilation of GOME ozone profiles and a global chemistry-transport model, Q. J. Roy. Meteor. Soc., 131, 477-502, 2005.

Simmons, A. J., Hortal, M., Kelly, G., et al.: ECMWF analyses and forecasts of stratospheric winter polar vortex breakup: September 2002 in the Southern Hemishere and related events, J. Atmos. Sci., 62, 668-689, 2005.

SPARC: Assessment of upper tropospheric and lower stratospheric water vapour, WCRP-113, WMO/TD No. 1043, SPARC Report No. 2, edited by: Kley, D., Russell, J. M., and Phillips, C., 2000.

Štajner, I., Benson, C., Liu, H.-C., et al.: Ice polar stratospheric clouds detected from assimilation of Atmospheric Infrared Sounder data, Geophys. Res. Lett., 34, L16802, doi:10.1029/2007GL029415, 2007.

Štajner, I., Riishøjgaard, L.-P., and Rood, R. B.: The GEOS ozone data assimilation system: Specification of error statistics, Q. J. Roy. Meteor. Soc., 127, 1069-1094, 2001.

Štajner, I. and Wargan, K.: Antarctic stratospheric ozone from the assimilation of occultation data, Geophys. Res. Lett., 31, L18108, doi:10.1029/2004GL020846, 2004.

Štajner, I., Wargan, K., Chang, L.-P., et al.: Assimilation of ozone profiles from the Improved Limb Atmospheric SpectrometerII: Study of Antarctic ozone, J. Geophys. Res., 111, D11514, doi:10.1029/2005JD006448, 2006.

Štajner, I., Winslow, N., Rood, R. B., and Pawson, S.: Monitoring of observation errors in the assimilation of satellite ozone data, J. Geophys. Res., 109, D06309, doi:10.1029/2003JD006309, 2004.

Stolarski, R. and Douglass, A. R.: Parameterization of the photochemistry of stratospheric ozone including catalytic processes, J. Geophys. Res., 90, 10709-10 718, 1985.

Streibel, M., Rex, M., von der Gathen, P., et al.: Chemical ozone loss in the Arctic winter 2002/2003 determined with Match, Atmos. Chem. Phys., 6, 2783-2792, 2006,

http://www.atmos-chem-phys.net/6/2783/2006/.

Struthers, H., Brugge, R., Lahoz, W. A., et al.: Assimilation of ozone profiles and total column measurements into a global general circulation model, J. Geophys. Res., 107, 4438, doi:10.1029/2001JD000957, 2002.

Susskind, J., Barnet, C., Blaisdell, J., et al.: Accuracy of geophysical parameters derived from Atmospheric Infrared Sounder/Advanced Microwave Sounding Unit as a function of fractional cloud cover, J. Geophys. Res., 111, D09S17, doi:10.1029/2005JD006272, 2006.
Swinbank, R., Ménard, R., and Riishøjgaard, L.-P.: Anisotropic error correlation modelling in a simple 2-D assimilation system. In Proceedings of the $3^{\text {rd }}$ International Symposium on assimilation of observations in meteorology and oceanography (Québec City, Canada 7-11 June 1999), World Weather Research Programme Report No. 2, WMO/TD No. 986, 353-356, 2000.

Talagrand, O.: Bayesian estimation. Optimal interpolation. Statistical linear estimation, in: Data Assimilation for the Earth System, NATO ASI Series, edited by: Swinbank, R., Shutyaev, V., and Lahoz, W. A., Kluwer, 21-35, 2003a.

Talagrand, O.: Variatonal assimilation. Adjoint equations, in: Data Assimilation for the Earth System, NATO ASI Series, edited by: Swinbank, R., Shutyaev, V., and Lahoz, W. A., Kluwer, 37-53, $2003 b$.

Talagrand, O.: A posteriori validation of assimilation algorithms, in: Data Assimilation for the Earth System, NATO ASI Series, edited by: Swinbank, R., Shutyaev, V., and Lahoz, W. A., Kluwer, 85-95, 2003c.

Tan, W. W., Geller, M. A., Pawson, S., and da Silva, A.: A case study of excessive subtropical transport in the stratosphere of a data assimilation system, J. Geophys. Res., 109, D11102, doi:10.1029/2003JD004057, 2004.

Trenberth, K. (Ed.): Climate System Modeling, Cambridge University Press, Cambridge, 788 pp., 1992.

Uppala, S. M., Kållberg, P., Simmons, A. J., et al.: The ERA-40 re-analysis, Q. J. Roy. Meteor. Soc., 131, 2961-3012, 2005.

van Loon, M., Builtjes, P. J. H. and Segers, A. J.: Data assimilation of ozone in the atmospheric transport chemistry model LOTUS, Environ. Modelling Software, 15, 603-609, 2000.

Vigouroux, C., De Mazière, M., Errera, Q., et al.: Comparison between ground-based FTIR and MIPAS $\mathrm{N}_{2} \mathrm{O}$ and $\mathrm{HNO}_{3}$ profiles before and after assimilation in BASCOE, Atmos. Chem. Phys., 7, 377-396, 2007, http://www.atmos-chem-phys.net/7/377/2007/.

Wang, K.-Y., Lary, D. J., Shallcross, D. E., et al.: A review on the use of the adjoint method in four-dimensional atmosphericchemistry data assimilation, Q. J. Roy. Meteor. Soc., 127, 21812204, 2001.

Wargan, K., Štajner, I., Pawson, S., et al.: Assimilation of ozone data from the Michelson Interferometer for Passive Atmospheric Sounding, Q. J. Roy. Meteor. Soc., 131, 2713-2734, 2005.

Waters, J. W.: Atmospheric measurements by the MLS experiments: Results from UARS and plans for the future, Adv. Space Res., 21, 1363-1372, 1998.

Waters, J. W., Froidevaux, L., Harwood, R. S., et al.: The Earth Observing System Microwave Limb Sounder (EOS MLS) on the Aura satellite, IEEE Trans. Geosci. Remote Sensing, 44, 10751092, 2006.

Weaver, A. T. and Courtier, P.: Correlation modelling on the sphere using a generalized diffusion equation, Q. J. Roy. Meteor. Soc., 127, 1815-1846, 2001.

WMO: Scientific Assessment of Ozone Depletion, 2006. World Meteorological Organization, Global Ozone Research and Monitoring Project, Report No. 50, available from http://www.wmo. ch/web/arep/reports/ozone_2006/ozone_asst_report.html, 2006.

Zupanski, D.: A general weak constraint applicable to operational 4DVAR data assimilation systems, Mon. Weather Rev., 125, 2274-2292, 1997. 